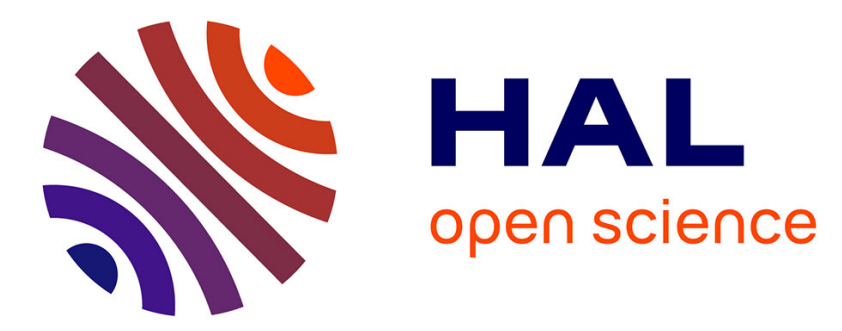

\title{
Parametric study of the mode coupling instability for a simple system with planar or rectilinear friction
}

\author{
Lucien Charroyer, Olivier Chiello, Jean-Jacques Sinou
}

\section{To cite this version:}

Lucien Charroyer, Olivier Chiello, Jean-Jacques Sinou. Parametric study of the mode coupling instability for a simple system with planar or rectilinear friction. Journal of Sound and Vibration, 2016, 384, pp. 94-112. 10.1016/j.jsv.2016.07.037 . hal-01380283

\section{HAL Id: hal-01380283 \\ https://hal.science/hal-01380283}

Submitted on 12 Oct 2016

HAL is a multi-disciplinary open access archive for the deposit and dissemination of scientific research documents, whether they are published or not. The documents may come from teaching and research institutions in France or abroad, or from public or private research centers.
L'archive ouverte pluridisciplinaire HAL, est destinée au dépôt et à la diffusion de documents scientifiques de niveau recherche, publiés ou non, émanant des établissements d'enseignement et de recherche français ou étrangers, des laboratoires publics ou privés. 


\title{
Parametric study of the mode coupling instability for a simple system with planar or rectilinear friction
}

\author{
L. Charroyer ${ }^{a, b, c}$, O. Chiello ${ }^{a, c}$, J-J. Sinou ${ }^{a, b, d}$ \\ ${ }^{a}$ Centre Lyonnais d'Acoustique, Université de Lyon, F-69000 Lyon, France. \\ ${ }^{b}$ Laboratoire de Tribologie et Dynamique des Systèmes UMR CNRS 5513, Ecole Centrale de Lyon, France. \\ ${ }^{c}$ IFSTTAR, AME, LAE, F-69500 Bron, France. \\ ${ }^{d}$ Institut Universitaire de France, 75005 Paris, France.
}

\begin{abstract}
In this paper, the study of a damped mass-spring system of three degrees of freedom with friction is proposed in order to highlight the differences in mode coupling instabilities between planar and rectilinear friction assumptions. Wellknown results on the effect of structural damping in the field of friction-induced vibration are extended to the specific case of a damped mechanical system with planar friction. It is emphasised that the lowering and smoothing effects are not so intuitive in this latter case. The stability analysis is performed by calculating the complex eigenvalues of the linearised system and by using the Routh-Hurwitz criterion. Parametric studies are carried out in order to evaluate the effects of various system parameters on stability. Special attention is paid to the understanding of the role of damping and the associated destabilisation paradox in mode-coupling instabilities with planar and rectilinear friction assumptions.
\end{abstract}

\section{Introduction}

Even if the problem of friction-induced vibrations has been a topic of great interest for many researchers in the past years $[1,2,3,4,5,6]$, squeal is still a current issue and a difficult subject since the effects of the system parameters are not completely understood. The comprehension of the phenomena involved in these vibrations and the choice of the contact models at the frictional interface have been much investigated $[2,3,4,7]$ and are still an active field of research. For example, Massi et al. [8] highlight the significant impact of both the contact surface topography and the frictional contact model. Many mechanisms have been proposed to explain the emergence of friction-induced instabilities. It is generally assumed that four categories of friction-induced vibration may be distinguished [4, 7]: stick-slip, variable dynamic friction coefficient, sprag-slip and mode coupling. Stick-slip was the first developed theory. Similarly, a decrease of the friction coefficient with relative sliding velocity (which introduces a negative damping due to tribological properties) can be chosen as the source of instability. In this case, a single structural mode can become unstable. Next, academic studies demonstrated that self-excited vibration can occur with a constant friction coefficient. Spurr [9] introduced the well-known sprag-slip phenomenon based on a geometrically induced instability. Then, several authors proposed the mode coupling mechanism as the origin of instability. In this latter case, the instability is the result of both the coupling of at least 
two structural modes of the mechanical system and the coupling between the normal and the tangential dynamics. It is associated to a Hopf bifurcation. This approach is now widely used to reproduce friction-induced vibrations in mechanical systems. Minimal models highlighting this type of instabilities can be easily found in the literature $[10,11,12]$. In most of these studies, the contact between the mass and the friction plane is modelled by a classical contact spring. It is a relatively common choice not only for this type of minimal models but also for most elaborated finite element models. This choice can be explained by physical considerations (i.e. local elasticity of the contact) or by numerical considerations (i.e. the friction force is directly linked to the normal displacement in order to simplify the problem formulation and the equations of motion). In addition, the authors often restrained the contact formulation at the frictional interface to one tangential degree-of-freedom (i.e. rectilinear friction). However, in this case, some specific phenomena due to the planar nature of the friction Coulomb law cannot be explained, particularly the fact that the direction of the friction force varies with the structure vibration.

A simple model combining these two characteristics (i.e. no contact spring and planar friction) was proposed by Moirot $[13,14]$. He outlined that differences between planar and rectilinear friction formulations can be highlighted and that destabilisation due to the planar friction may occur. However, Moirot's study focused only on a system without damping. On the contrary, similar studies carried out on more complex finite element models $[15,16,17]$ showed that planar friction seems to stabilise the system.

Other planar effects of friction have already been investigated in the general context of friction-induced vibrations or brake squeal. Kinkaid et al. [18] proposed a new mechanism for disc brake noise by taking into account both longitudinal and transverse braking directions for a 4-dof model. They demonstrated that instabilities in the radial vibration can be observed during the transient processes. Oberst and Lai [19] also studied nonlinear friction coupling in disc brake squeal and more specifically the influence of the lateral vibration of a 2-dof friction oscillator. Considering the variation of the belt angle, they showed that a perturbation in the radial component in a brake system can also cause instabilities. Zhang et al. [20] recently studied the stability of coupled friction oscillators on sliding rigid plate with planar friction. They investigated the influence of the uncertainty arising from the tribological aspect and proposed different types of friction modelling with randomised parameters.

However, the precise role of planar friction on mode-coupling instabilities, compared with rectilinear friction, remains an open question. The objective of the present study is to investigate this scientific issue thoroughly. One of the most interesting phenomena concerns the existence of a non-intuitive effect of damping distribution that can generate unstable vibrations. As previously reported in many recent works, structural damping is of primary importance to mode coupling stability and results indicate that the addition of damping in mechanical systems alters the stable-unstable boundaries. In the literature, the relationship between damping and system propensity to develop instability and the so-called "destabilisation paradox" applied to friction-induced vibration have often been illustrated by several analytical and numerical studies [12, 21, 22, 23]. Many contributions on the destabilisation paradox in conservative systems with damping have also been carried out in a general context by Kirillov [24, 25, 26, 27]. He showed that the effect of proportional and nonproportional damping on the reversible Hopf bifurcation in systems with rectilinear friction is in qualitative agreement with the general theory of the destabilisation paradox in circulatory systems [25, 26, 27]. Massi and Giannini [28] also proposed an experimental investigation of the relationship between the distribution of modal damping and the propensity 
to develop squeal in a beam-on-disk setup. They highlighted that a nonuniform repartition of the modal damping causes an increase of the squeal propensity. Extensive studies have also been conducted including the role of damping with other physical factors. For example Hervé et al. [29, 30] used a two-degree-of-freedom nonlinear model of clutch squeal in order to examine in detail the influence of structural damping on the effects of the combined circulatory and gyroscopic actions. Recently, Kirillov et al. [25, 26, 27] worked on the determination in an explicit form of the stabilising damping configurations for a large class of non-conservative systems. They proposed a theory for the qualitative and quantitative description of the destabilisation paradox in such systems. In the case of a vibrational system, planar friction introduces a new contribution of damping which has not been examined in this perspective. The additional inclusion of structural damping also remains an open question. Thus, an original contribution of the present study is to extend well-known results on the non-intuitive effects of damping distribution for the specific case of a damped mechanical system with planar friction.

First, the mechanical system under study, the background on stability analysis and the Routh-Hurwitz criterion are presented. Second, parametric studies and numerical results for damped and undamped systems with planar or rectilinear friction are investigated in order to discuss the elementary effect of a planar or rectilinear friction and to undertake the extension of the destabilisation paradox in the presence of planar friction.

\section{Description of the mechanical model}

The system considered in this study is described in Figure 1. It is composed of a mass $m$ in frictional contact with a rigid plane moving with a constant rectilinear velocity. The value of the imposed velocity is denoted $V$ while its direction is denoted $\vec{t}$ as indicated in Figure 1. The mass is held against the moving plane by three springs and pressed by an external force $F$. Damping is also included as shown in Figure 1. The angle between the direction $\vec{t}$ of the imposed velocity of the rigid plane and the coordinate $\vec{x}$ of the mechanical system is defined by $\theta$. The equations of motion can be written in matrix form:

$$
\mathbf{M U}+\mathbf{C} \dot{U}+\mathbf{K U}=\mathbf{R}+\mathbf{F}
$$

where $\mathbf{U}=\left(\begin{array}{lll}U_{x} & U_{y} & U_{z}\end{array}\right)^{T}, \dot{\mathbf{U}}$ and $\ddot{\mathbf{U}}$ denote respectively the displacement, velocity and acceleration vectors along the three directions $x, y$ and $z$. The mass matrix $\mathbf{M}$ is defined by $\mathbf{M}=m \mathbf{I}$ (with $\mathbf{I}$ the identity matrix). The vector $\mathbf{F}$ represents the external force. The vector $\mathbf{R}$ includes the contact normal reaction and the friction force applied on the centre of mass due to frictional contact with the plane. $\mathbf{C}$ and $\mathbf{K}$ are respectively the damping and stiffness matrices. They are given by :

$$
\begin{gathered}
\mathbf{C}=\left[\begin{array}{ccc}
c_{x} & 0 & 0 \\
0 & c_{y} & 0 \\
0 & 0 & c_{z}
\end{array}\right] \\
\mathbf{K}=\left[\begin{array}{ccc}
k_{1} & 0 & k_{1} \tan \alpha \\
0 & k_{2} & k_{2} \tan \beta \\
k_{1} \tan \alpha & k_{2} \tan \beta & k_{n}
\end{array}\right] \\
\mathbf{F}=\left(\begin{array}{lll}
0 & 0 & -F_{n}
\end{array}\right)^{T} \quad \text { with } F_{n}>0
\end{gathered}
$$


with

$$
\begin{gathered}
k_{1}=k_{1}^{\prime} \cos ^{2} \alpha \\
k_{2}=k_{2}^{\prime} \cos ^{2} \beta \\
k_{n}=k_{n}^{\prime}+k_{1} \tan ^{2} \alpha+k_{2} \tan ^{2} \beta
\end{gathered}
$$

The coupling between the normal and the tangential degrees-of-freedom is induced by angles $\alpha$ and $\beta$. It is a necessary condition for the occurrence of mode coupling instability. The choice of orthogonal springs $k_{1}^{\prime}$ and $k_{2}^{\prime}$ is only introduced to simplify the formulation of the dynamic equation of the system and to make the comprehension of various phenomena on the instability occurrence easier. It provides uncoupling equations for the tangential degrees-of-freedom (i.e. the two modes concerned by the mode coupling phenomena) without friction. This choice does not limit the results generality, since other configurations can be found with an appropriate modification of the coordinate system. For the readers' comprehension, there is no geometrical symmetry in the $(x, y)$ plane if stiffness $k_{1}$ and $k_{2}$ or angles $\alpha$ and $\beta$ are different.

In this study, we are interested in the occurrence of instability around the sliding equilibrium (i.e. the appearances of self-excited system oscillations due to mode coupling). The contact is assumed to be bilateral and sliding: the mass remains in contact with the plane and the mass tangential velocity remains lower than the velocity of the rigid plane so that no adhesion can occur. The friction force is given by the sliding part of the Coulomb's law with a constant coefficient $\mu$. The contact equations can be expressed as :

$$
\left\{\begin{aligned}
\mathbf{R} & =\mathbf{P}_{\mathbf{t}}^{T} R_{t}+\mathbf{P}_{\mathbf{b}}{ }^{T} R_{b}+\mathbf{P}_{\mathbf{n}}{ }^{T} R_{n} \\
U_{z} & =0 \\
\left\{\begin{array}{l}
R_{t} \\
R_{b}
\end{array}\right\} & =\mu R_{n} \frac{\left\{\begin{array}{c}
V-\dot{U}_{t} \\
-\dot{U}_{b}
\end{array}\right\}}{\left\|\left\{\begin{array}{c}
V-\dot{U}_{t} \\
-\dot{U}_{b}
\end{array}\right\}\right\|}
\end{aligned}\right.
$$

where

$$
\begin{gathered}
\mathbf{P}_{\mathbf{b}}=\left[\begin{array}{lll}
-\sin \theta & \cos \theta & 0
\end{array}\right] \\
\mathbf{P}_{\mathbf{t}}=\left[\begin{array}{lll}
\cos \theta & \sin \theta & 0
\end{array}\right] \\
\mathbf{P}_{\mathbf{n}}=\left[\begin{array}{lll}
0 & 0 & 1
\end{array}\right]
\end{gathered}
$$

$R_{t}, R_{b}$ and $R_{n}$ are respectively the two components of the friction force and the contact reaction which is applied to the mass, according to the directions $\vec{t}, \vec{b}$ and $\vec{z}$. $\dot{U}_{t}=\mathbf{P}_{\mathbf{t}} \dot{\mathbf{U}}$ and $\dot{U}_{b}=\mathbf{P}_{\mathbf{b}} \dot{\mathbf{U}}$ are the mass velocities according to the directions $\vec{t}$ and $\vec{b}$. Matrices $\mathbf{P}_{\mathbf{t}}, \mathbf{P}_{\mathbf{b}}$ and $\mathbf{P}_{\mathbf{n}}$ are used to transpose forces from the contact to the global coordinate system. For rectilinear friction, the friction force component in direction $\vec{b}$ is zero and Coulomb's law in the sliding phase is simply written $R_{t}=\mu R_{n}$, which is directly linear. For planar friction, equation (8) remains non-linear and has to be linearised in order to achieve the stability analysis.

[Figure 1 about here.] 


\section{Stability analysis}

In this section, the stability of the system is studied. It consists in analysing the behaviour of small perturbations around the quasi-static sliding equilibrium. In this purpose, the calculation of this equilibrium is firstly explained. Then, the friction equations are linearised around the equilibrium and the problem is projected on the tangential plane. Finally the stability of the equilibrium is determined by the classical Complex Eigenvalues Analysis (CEA) or by the Routh-Hurwitz criterion.

\subsection{Calculation of the equilibrium}

First, the equilibrium $\mathbf{U}^{\mathbf{e}}$, such that $\ddot{\mathbf{U}}^{\mathbf{e}}=0$ and $\dot{\mathbf{U}}^{\mathbf{e}}=0$, is calculated. Since the tangential velocities of the mass are zero, we have :

$$
\left\{\begin{array}{l}
R_{t}^{e}=\mu R_{n}^{e} \\
R_{b}^{e}=0
\end{array}\right.
$$

where $R_{t}^{e}$ and $R_{b}^{e}$ are the quasi-static frictions forces according to directions $\vec{t}$ and $\vec{b}$ respectively and $R_{n}^{e}$ is the quasi-static normal reaction. We now have to solve the following system:

$$
\left\{\begin{aligned}
\mathbf{K} \mathbf{U}^{\mathbf{e}} & =\mathbf{F}+\left(\mu \mathbf{P}_{\mathbf{t}}+\mathbf{P}_{\mathbf{n}}\right) R_{n}^{e} \\
U_{z}^{e} & =0
\end{aligned}\right.
$$

which leads to the following equations :

$$
\left\{\begin{aligned}
\mathbf{U}^{\mathbf{e}} & =\mu R_{n}^{e}\left(\begin{array}{lll}
\frac{\cos \theta}{k_{1}} & \frac{\sin \theta}{k_{2}} & 0
\end{array}\right)^{T} \\
R_{n}^{e} & =\frac{F_{n}}{1-\mu(\cos \theta \tan \alpha+\sin \theta \tan \beta)}
\end{aligned}\right.
$$

The last equation of system (14) explains the relation between $R_{n}^{e}$ and the system factors and requires that $R_{n}^{e}>0$ to ensure the contact. Therefore, recalling that $F_{n}>0$, a critical friction coefficient $\mu^{e}$ below which the quasi-static sliding equilibrium exits is given by :

$$
\mu^{e}=\frac{1}{\cos \theta \tan \alpha+\sin \theta \tan \beta}
$$

We notice that for the proposed model, only the angles $\alpha, \beta$ and the friction direction $\theta$ have an influence on the equilibrium existence. 


\subsection{Stability around the equilibrium}

The stability of the system is studied for small perturbations $\delta \mathbf{U}$ around the equilibrium $\mathbf{U}^{\mathrm{e}}$ of the system (i.e. $\delta \mathbf{U}+\mathbf{U}^{\mathbf{e}}$ ). By linearising the third equation of system (8), the following equation is obtained:

$$
\begin{aligned}
\left\{\begin{array}{l}
\delta R_{t} \\
\delta R_{b}
\end{array}\right\} & =\left.\frac{\partial\left\{\begin{array}{l}
R_{t} \\
R_{b}
\end{array}\right\}}{\partial R_{n}}\right|_{\dot{\mathbf{U}}=\mathbf{0}, R_{n}=R_{n}^{e}} \delta R_{n}+\left.\frac{\partial\left\{\begin{array}{l}
R_{t} \\
R_{b}
\end{array}\right\}}{\partial \dot{\mathbf{U}}}\right|_{\dot{\mathbf{U}}=\mathbf{0}, R_{n}=R_{n}^{e}} \delta \dot{\mathbf{U}} \\
& \left.=\mu\left\{\begin{array}{l}
1 \\
0
\end{array}\right\} \delta R_{n}-\frac{\mu R_{n}^{e}}{V}\left[\begin{array}{ll}
1 & 0 \\
0 & 1
\end{array}\right]-\left\{\begin{array}{l}
1 \\
0
\end{array}\right\} \otimes\left\{\begin{array}{l}
1 \\
0
\end{array}\right\}\right]\left\{\begin{array}{l}
\delta \dot{U}_{t} \\
\delta \dot{U}_{b}
\end{array}\right\} \\
& =\left\{\begin{array}{c}
\mu \delta R_{n} \\
-\mu \frac{R_{n}^{e}}{V} \delta \dot{U}_{b}
\end{array}\right\}
\end{aligned}
$$

In the case of rectilinear friction, component $\delta R_{b}$ is neglected and Coulomb's law is directly written again. With planar friction, the term $-\mu \frac{R_{n}^{e}}{V} \delta \dot{U}_{b}$, resulting from the linearisation of the friction force direction, provides a velocity contribution. This additional term, occurring only in the case of planar friction, corresponds to a new damping contribution characterised by the viscous damping coefficient $c_{b}=\frac{R_{n}^{e}}{V}$ and depending on the sliding equilibrium. This contribution, which increases when the external force increases or the sliding velocity decreases, can be taken into account through a damping matrix $\mathbf{C}_{\mathbf{b}}$. The system dynamic is now given by :

$$
\left\{\begin{aligned}
m \delta \ddot{\mathbf{U}}+\left(\mathbf{C}+\mathbf{C}_{\mathbf{b}}\right) \delta \dot{\mathbf{U}}+\mathbf{K} \delta \mathbf{U} & =\left(\mu \mathbf{P}_{\mathbf{t}}+\mathbf{P}_{\mathbf{n}}\right) \delta R_{n} \\
\delta U_{z} & =0
\end{aligned}\right.
$$

with

$$
\mathbf{C}_{\mathbf{b}}=\mu \mathbf{P}_{\mathbf{b}}{ }^{T} \frac{R_{n}^{e}}{V} \mathbf{P}_{\mathbf{b}}
$$

Finally, a unique formulation using displacements, velocities and accelerations in the tangential plane can be obtained by substituting the normal reaction in the problem. The first step uses the fact that the normal displacement is zero for expressing $\delta R_{n}$ according to $\mathbf{U}_{\mathbf{p}}=\left(\begin{array}{ll}\delta U_{x} & \delta U_{y}\end{array}\right)^{T}$ :

$$
\delta R_{n}=\left[\begin{array}{ll}
k_{1} \tan \alpha & k_{2} \tan \beta
\end{array}\right] \mathbf{U}_{\mathbf{p}}
$$

Then, by using this equation, the motion equation projected into the tangential plane can be written as:

$$
m \ddot{\mathbf{U}}_{\mathbf{p}}+\left(\tilde{\mathbf{C}}+\tilde{\mathbf{C}}_{\mathbf{b}}\right) \dot{\mathbf{U}}_{\mathbf{p}}+\left(\tilde{\mathbf{K}}+\mathbf{K}_{\mu}\right) \mathbf{U}_{\mathbf{p}}=0
$$

where $\tilde{\mathbf{C}}, \tilde{\mathbf{C}}_{\mathbf{b}}$ and $\tilde{\mathbf{K}}$ are the restrictions of the matrices $\mathbf{C}, \mathbf{C}_{\mathbf{b}}$ and $\mathbf{K}$ to the tangential plane, respectively. Matrices $m \tilde{\mathbf{I}}$, $\tilde{\mathbf{C}}$ and $\tilde{\mathbf{K}}$ are diagonal. They characterise the two uncoupled modes which determine the sliding system behaviour without friction. Moreover, we have :

$$
\mathbf{K}_{\mu}=-\mu \tilde{\mathbf{P}}_{\mathbf{t}}^{T}\left[\begin{array}{ll}
k_{1} \tan \alpha & k_{2} \tan \beta
\end{array}\right]
$$

where $\tilde{\mathbf{P}}_{\mathbf{t}}=\left[\begin{array}{ll}\cos \theta & \sin \theta\end{array}\right]$ is the restriction of $\mathbf{P}_{\mathbf{t}}$ to the tangential plane. The friction effect is linked to matrices $\tilde{\mathbf{C}}_{\mathbf{b}}$ and $\mathbf{K}_{\mu}$ which lead to mode coupling. It can be noted that the matrix $\tilde{\mathbf{C}}_{\mathbf{b}}$ is symmetric while matrix $\mathbf{K}_{\mu}$ is non-symmetric. 
Furthermore, mode coupling depends on the system geometry with an inherent normal/tangential coupling due to angles $\alpha$ and $\beta$ and on the friction direction in relation to the system which is characterised by the angle $\theta$.

The system stability is analysed by calculating the complex eigenvalues $\lambda$ of the characteristic equation :

$$
\operatorname{det}\left(\lambda^{2} m \tilde{\mathbf{I}}+\lambda\left(\tilde{\mathbf{C}}+\tilde{\mathbf{C}}_{\mathbf{b}}\right)+\left(\tilde{\mathbf{K}}+\mathbf{K}_{\mu}\right)\right)=0
$$

If all the real parts of the complex eigenvalues are negative, then the system equilibrium is stable. However if, one real part becomes positive, the equilibrium becomes unstable.

To determine the stability areas of the system equilibrium according to different factors, the Routh-Hurwitz criterion can also be used [31]. One of the main advantages of the Routh-Hurwitz criterion is to provide analytical expressions versus the stability analysis of the mechanical system under study. The development of equation (22) yields to a polynomial equation of degree 4 :

$$
\lambda^{4}+a_{3} \lambda^{3}+a_{2} \lambda^{2}+a_{1} \lambda+a_{0}=0
$$

where $\lambda$ are the eigenvalues of the linearised mechanical system. The application of Routh-Hurwitz criterion to this characteristic equation gives the four following coefficients $a, b, c$ and $d$

$$
\begin{gathered}
a=a_{0} \\
b=a_{3} \\
c=a_{2} a_{3}-a_{1} \\
d=a_{1}\left(a_{2} a_{3}-a_{1}\right)-a_{0} a_{3}^{2}
\end{gathered}
$$

Generally speaking, if all these coefficients are positive, the equilibrium is stable. When at least one of the four coefficients is negative, the equilibrium is unstable. In our physical practical case, it may be observed that $a>0, b>0$ and $c>0$. Thus, equilibrium stability is governed only by coefficient $d$ : if $d>0$, then the equilibrium is stable (i.e. $\forall i \Re\left(\lambda_{i}\right)<0$ ). If $d<0$ the equilibrium is unstable. By using the Routh-Hurwitz criterion, the critical friction coefficients $\mu_{c}$ such that $d\left(\mu_{c}\right)=0$ can be determined. The stability of the equilibrium changes when $\mu=\mu_{c}$. Depending on different cases, the critical friction coefficient are solutions of a polynomial equation of degree 1 (planar friction without damping), degree 2 (rectilinear friction with or without damping) or degree 4 (planar friction with damping). In the last two cases, if there are two positive solutions, the equilibrium can become stable again after being unstable. All the mathematical developments and results are given in Appendix A.

In the following section, the stability of the mechanical system will be analysed by calculating the complex eigenvalues $\lambda$ of the characteristic equation defined in equation 22.

\section{Results}

This paper presents the results for two specific cases. The first one concerns the undamped model. It not only provides an overview of the influence of some physical parameters but also offers the opportunity to discuss the elementary effect of a planar or rectilinear friction. The second case concerns the damped model and undertake the extension of the destabilisation paradox in the presence of planar friction. 
The study is performed for a reference undamped system with the following parameters : $m=0.01 \mathrm{~kg}, k_{i}=m \omega_{i}^{2}$ for $i=1,2$ with $\omega_{1}=100 \times 2 \pi \mathrm{rad} \mathrm{s}^{-1}, \omega_{2}=75 \times 2 \pi \mathrm{rad} . \mathrm{s}^{-1}, \alpha=30^{\circ}, \beta=60^{\circ}, F_{n}=10 \mathrm{~N}, \theta=-30^{\circ}$ and $V=5 \mathrm{~m} . \mathrm{s}^{-1}$. Parameters differing from these reference values and addition of damping will be explicitly specified.

\subsection{Undamped model with planar or rectilinear friction: parameter influence on stability area and complex eigenvalues}

In this section a parametric study on the stability of the undamped system is carried out. The influence of the equilibrium, the system stiffness and the sliding direction on the stability area (i.e. the boundary between the stable and unstable zones) are treated.

\subsubsection{Stability area: presentation and understanding}

This paragraph introduces the graphical representation of the stability areas used in the paper. It also gives first results showing possible differences in the system stability depending on whether planar or rectilinear friction is considered.

Figure 2 shows the difference between planar and rectilinear friction in the stability area of the undamped system. This graphic has two variable parameters : the direction of the sliding velocity $\theta$ and the friction coefficient $\mu$. For each couple $(\theta, \mu)$, the boundary between the stable and unstable zones is determined (in blue for rectilinear friction and in red for planar friction). In addition, all the stable and unstable zones are labelled with the wording "stable" or "unstable" in blue (rectilinear friction) or red (planar friction). For friction coefficients $\mu>\mu_{e}(\theta)$ (see equation (15)), there is no sliding equilibrium: this is indicated by "No equilibrium" in green. To properly understand the reading of this graph, let us illustrate with two examples. At first we consider the couple $\left(-45^{\circ}, 0.5\right)$ indicated by a circle in Figure 2. It is observed that the mechanical system is unstable in both friction cases. In a second example, we consider the couple $\left(-10^{\circ}, 0.2\right)$ marked with a star in Figure 2. In this case, the system with planar friction is unstable whereas the system with rectilinear friction is stable.

More generally, regarding the stable area versus all the possible couples $(\theta, \mu)$, it can be noticed that the undamped system is less stable with planar friction than with rectilinear friction. We can also observed that the boundary of instability is not symmetric with respect to the diagonal line defined by $\theta=-45^{\circ}$. This is not surprising since there is no geometrical symmetry in the system (i.e. the stiffness $k_{1}$ and $k_{2}$ as well as the angles $\alpha$ and $\beta$ are different).

[Figure 2 about here.]

\subsubsection{Influence of stiffness}

In this section, the influence of the stiffness is studied. The stiffness sum is fixed using reference value $k_{1}+k_{2}=$ $156.25 \times(2 \pi)^{2} \mathrm{~N} \cdot \mathrm{m}^{-1}$ whereas three values of stiffness difference are selected: $k_{1}-k_{2}=8.75 \times(2 \pi)^{2} \mathrm{~N} \cdot \mathrm{m}^{-1}$, $43.75 \times(2 \pi)^{2} \mathrm{~N} \cdot \mathrm{m}^{-1}$ and $78.75 \times(2 \pi)^{2} \mathrm{~N} \cdot \mathrm{m}^{-1}$, the middle one corresponding to the reference case. Figures 3(a) and (b) show the stability areas for the system with planar friction and rectilinear friction respectively. At $\theta=-30^{\circ}$, the Hopf bifurcation (i.e. the variation of the real part of the eigenvalues with the friction coefficient) and the variation of the frequencies are represented in Figures 3(c), (d), (e) and (f) for both planar and rectilinear friction. 
Figures 3(a) and (b) point out that the difference between $k_{1}$ and $k_{2}$ has a real impact on the system stability. The stable and unstable areas can drastically change according to variations in these parameters. The effect is rather similar with planar or rectilinear friction. We observe that the lower the stiffness difference, the less stable the system equilibrium (i.e. the stable area increases on the graph versus the couple $(\theta, \mu)$ ). This can be easily explained by the fact that the difference between the mode frequencies that can coalesce is directly linked to the difference between the stiffness $k_{1}$ and $k_{2}$ : if the difference between $k_{1}$ and $k_{2}$ is low, the difference between the frequencies is also low and coalescence between the associated modes is easier. Thereby, mode coupling instabilities appear at a lower friction coefficient $\mu$ as illustrated in Figures 3(c) and (d).

Figures 3(c), (d), (e) and (f) clearly illustrate the difference between planar and rectilinear friction. In the case of rectilinear friction, the classical behaviour of an undamped system is obtained (see Figures 3(d) and (f)). As long as the system is stable, the real parts are equal to zero. Once the system becomes unstable, real part variations versus the friction coefficient are symmetrical with respect to the real part axis (i.e. symmetrical about zero) and a perfect coalescence pattern is obtained (meaning that the frequencies of the unstable mode and the associated stable mode are exactly the same, as previously indicated in [12, 21, 23, 32]). In contrast, in the case of planar friction, the coalescence pattern is not perfect (the unstable mode and the associated stable mode have not the same frequency in Figure 3(c)). Likewise, in Figure 3(c), the real part variations are more complex due to the additional damping term provided by planar friction (see Section 3.2). Table 1 compares the friction coefficients $\mu_{c}$ at the Hopf bifurcation point and the frequencies of the unstable mode for planar or rectilinear friction. It turns out that planar friction also induces a modification of the Hopf bifurcation point.

[Table 1 about here.]

[Figure 3 about here.]

\subsubsection{Influence of sliding velocity}

The influence of the sliding velocity on the stability of the undamped system with planar friction is now studied. As previously seen in Section 3.2, the consideration of planar friction provides an additional damping term (i.e. matrix $\tilde{\mathbf{C}}_{\mathbf{b}}$ ) resulting from the linearisation of the friction force direction. The amplitude of this term depends on sliding velocity $V$. In the case of rectilinear friction, this contribution does not exist. To determine the influence of this damping contribution on the system stability, a complex eigenvalue analysis is carried out using three values of the sliding velocity $V=1$ $\mathrm{m} . \mathrm{s}^{-1}, 5 \mathrm{~m} \cdot \mathrm{s}^{-1}$ and $10 \mathrm{~m} \cdot \mathrm{s}^{-1}$. For conciseness, the stability areas are not shown and only the eigenvalues graphs for $\theta=-30^{\circ}$ are presented. Indeed, the variation of the sliding velocity has no effect on the stability areas (i.e. $\mu_{c}$ does not depend on $c_{b}$ as shown by the expression of coefficient $d(\mu)$ in section A.2 of appendix A). Results on the boundaries of stable and unstable zones are identical to those presented in the previous section 4.1.1 (Figures 2).

Figures 4(a) and (b) display the variations of the real and imaginary parts for $\theta=-30^{\circ}$ respectively (versus the friction coefficient for the three values of the sliding velocity). First of all, Figure 4(a) shows that the system becomes unstable at a lower friction coefficient with planar friction than with rectilinear friction. Thus, the consideration of planar friction

can contribute to the destabilisation paradox: an increase in damping via the additional damping contribution $\tilde{\mathbf{C}}_{\mathbf{b}}$ may tend to make the system unstable for a lower value. As already mentioned, the sliding velocity has no influence on the 
stability boundary: in each planar friction configuration, the system becomes unstable for the same values of the friction coefficient at Hopf bifurcation point $\mu_{c}$ (see Figure 4(a) for $\mu=\mu_{c}=0.168$ and Table 2). However, the sliding velocity drastically affects the real part variations. Different behaviours can be observed according to the friction coefficient range. For $\mu$ in $\left[0 ; \mu_{c}\right]$, a decrease of the sliding velocity lowers the real part. The same behaviour is observed for higher friction coefficients (i.e. $\mu>0.25$ ). However for friction coefficients in $\left[\mu_{c} ; 0.25\right]$, a decrease of the sliding velocity increases the

value of the real part. In the whole range, when $V$ is large (i.e. $c_{b}=\frac{R_{n}^{e}}{V}$ is small), the behaviour of the system tends to the rectilinear friction case.

Finally, Figure 4(b) shows the variation of the associated frequencies according to the friction coefficient $\mu$ for the three values of the sliding velocity. A decrease of the sliding velocity $V$ leads to a damping increase provided by planar friction and has an influence on mode coalescence. A decrease of the sliding velocity increases the phenomenon of imperfect coalescence. This is notably true at the Hopf bifurcation point as indicated in Table 2. This is a direct consequence of the influence of damping (and more precisely the "smoothing effect") and supports existing studies on the subject $[10,12,23,29]$.

\section{[Figure 4 about here.]}

[Table 2 about here.]

\subsubsection{Influence of sliding velocity direction}

The influence of the sliding velocity direction is studied more deeply in this section. The variations of the real parts, the frequencies and the complex plane are displayed for two directions $\left(\theta=-30^{\circ}\right.$ and $\left.-60^{\circ}\right)$. The sliding velocity is equal to $5 \mathrm{~m} . \mathrm{s}^{-1}$ in both cases. For the reader's comprehension, it is recalled that the stability areas (i.e. the boundaries between stable and unstable zones) for the undamped system with planar friction (and the direction of the sliding velocity between $-180^{\circ}$ and $180^{\circ}$ ) are given in Section 4.1.1.

Figure 5(a) shows that the sliding velocity direction affects the system stability: for $\theta=-30^{\circ}$, the real parts of the eigenvalues become positive beyond a lower friction coefficient than for $\theta=-60^{\circ}$. Figure $5(\mathrm{~b})$ shows the frequency variations and outlines the occurrence of a crossing of both modes involved in the coalescence pattern for $\theta=-60^{\circ}$.

Finally, one major effect of the sliding velocity direction can be seen in Figure 5(c) which depicts the variation of the modes in the complex plane (variations of the real and imaginary parts of eigenvalues). For $\theta=-30^{\circ}$, the unstable mode at the Hopf bifurcation point has the higher frequency (i.e. the mode with a frequency of $100 \mathrm{~Hz}$ for $\mu=0$ ) whereas the mode with the lower frequency corresponds to the associated stable mode. On the contrary, for $\theta=-60^{\circ}$, the unstable mode at the Hopf bifurcation point corresponds to the mode with the lower frequency (the mode with a frequency of $75 \mathrm{~Hz}$ for $\mu=0$ ) and beyond the mode coalescence, the crossing of the stable and unstable modes causes the unstable mode to have higher frequency. This phenomenon is proper to planar friction. In the case of rectilinear friction, the unstable mode corresponds to the mode with the higher frequency for both values of $\theta$.

[Figure 5 about here.] 


\subsection{Influence of damping for a system with planar or rectilinear friction}

One main original contribution of this section is to extend well-known results proposed by many of researchers on the effect of structural damping in the field of friction-induced noise and vibration by considering the specific case of a system with planar friction. It will illustrate that the "lowering effect" (that tends to stabilise the mechanical system with rectilinear friction if the two modes involved in the coalescence are equally damped) and the "smoothing effect" (that may act in an unintuitive way by destabilising the system $[10,12,23])$ are significantly affected by planar friction. To achieve this goal, the influence of damping on system stability is analysed for planar or rectilinear friction. Comparisons with results of Section 4.1 (i.e. undamped system with planar or rectilinear friction) and differences in the stability of the damped system depending on friction assumption will be undertaken. Damping is defined by default as $\eta=0.02$ and $c=m \eta \omega_{1}$. Firstly, the influence of an iso-damping $\left(\eta_{1}=\eta_{2}\right)$ is studied for the mechanical system with planar or rectilinear friction. Effects of a non-iso-damping is discussed then. Finally, the influence of the sliding velocity for the damped system is highlighted.

\subsubsection{Influence of iso-damping}

A stability analysis of the iso-damped system is carried out for three different damping coefficients values: $\eta=0.01,0.02$ and 0.05. Figures 6(a) and (b) depict the stability areas for planar friction and a rectilinear friction, respectively. Firstly, we observe that the boundaries between the stable and unstable zones for the damped system with planar or rectilinear friction are quite close even if some differences can appear. For the undamped system, the results on the stability areas obtained with planar or rectilinear friction were very different (see Section 4.1). Thus, the contribution of a structural isodamping in the system completely changes the previous comments about the impact of the choice of friction. Moreover, the extent of the stable zones increases with damping $\eta$ for all the values of $\theta$ between $[0 ;-90]^{\circ}$. This reflects the fact that adding proportional damping stabilises the system for both planar and rectilinear frictions.

To deepen the influence of iso-damping, the variation of the real and imaginary parts of the eigenvalues are plotted in Figures 6(c) and (e), and Figures (d) and (f) for planar and rectilinear frictions respectively and $\theta=-30^{\circ}$. First of all, as previously explained by some authors $[10,12,23]$ for systems with rectilinear friction, increasing damping $\eta$ makes the eigenvalue real parts decrease, whereas the frequencies remain approximately constant, as illustrated in Figures6(d) and (f). In addition, since the two modes involved in the coalescence are equally damped, the coalescence pattern is perfect. This phenomenon of iso-damping, called the lowering effect, extends to the mechanical system with planar friction : increasing $\eta$ enlarges the stability range since the real parts are lowered evenly and become positive for a higher value of the friction coefficient, as indicated in Figure 6(c). Likewise, changes in frequencies are invariant with respect to isodamping $\eta$. The imperfect coalescence of modes is only due to the presence of the additional damping term $\tilde{\mathbf{C}}_{\mathbf{b}}$ that is provided by planar friction. Table 3 assesses the friction coefficient at the Hopf bifurcation point and the frequency of the unstable mode for the different cases.

Thus, the only noticeable difference between rectilinear and planar friction seems to be the sensibility of the boundaries between the stable and unstable areas: an increase of iso-damping $\eta$ has a larger effect on the stability area with planar friction (see Figures 6(a) and (b)). This can easily be explained by considering the slope of the real parts versus the friction coefficient. In the case of planar friction, the slope of the real part is lower due to the presence of the additional damping 
term $\tilde{\mathbf{C}_{\mathbf{b}}}$. This leads to a stronger modification of Hopf bifurcation point $\mu_{c}$ in comparison with rectilinear friction, as illustrated in Figures 6(c) and (d). However it must be recalled that the additional damping term due to planar friction can also contribute to the destabilisation paradox and may tend to make the system unstable for a lower value of the friction coefficient than with rectilinear friction as previously shown in Section 4.1.3.

In conclusion, due to the addition of iso-damping, the stability areas obtained with planar friction are close to those obtained with rectilinear friction. This can be globally explained by the fact that the added damping matrix $\tilde{\mathbf{C}}$ (i.e. contribution of iso-damping) limits the effects of the contribution of matrix $\tilde{\mathbf{C}}_{\mathbf{b}}$ due to planar friction. We recall, however, that these results obviously depend on the set of physical parameters. If the contributions of matrices $\tilde{\mathbf{C}}_{\mathbf{b}}$ and $\tilde{\mathbf{C}}$ are changed and the contribution of $\tilde{\mathbf{C}}_{\mathbf{b}}$ becomes much more important than that of $\tilde{\mathbf{C}}$ (e.g. by changing sliding velocity $V$ or iso-damping $\eta$ ), larger differences between planar and rectilinear friction may occur (as described in Section 4.1.1).

[Table 3 about here.]

[Figure 6 about here.]

\subsubsection{Influence of non-proportional damping}

The influence of a non-proportional damping is now studied for planar and rectilinear friction. One main contribution of this section is to investigate the extension of the well-known destabilisation paradox due to the presence of nonproportional damping and the associated phenomena such as the lowering and smoothing effects to the specific case of a system with planar friction. The stability areas are plotted for several values of $\eta_{x}$ with $\eta_{y}=0.02$ constant such as $\frac{\eta_{y}}{\eta_{x}}=0.5,1.0$ and 2.0 and for several values of $\eta_{y}$ with $\eta_{x}=0.02$ constant such as $\frac{\eta_{x}}{\eta_{y}}=0.5,1.0$ and 2.0. Figures 7 (a) and (c) show the stability areas for planar friction whereas Figures 7(b) and (d) correspond to the stability areas for rectilinear friction. The stability areas for planar or rectilinear friction and several distributions of non-proportional damping are quite close even if some differences are noticeable. For example, in the case of planar friction, it is noticed that the variation of $\eta_{x}$ or $\eta_{y}$ has some effect on stability for sliding velocity directions close to $\mathrm{x}$-axis $\left(0^{\circ}\right.$ or $\left.180^{\circ}\right)$ or to $\mathrm{y}$-axis $\left(90^{\circ}\right.$ or $\left.-90^{\circ}\right)$. In the case with rectilinear friction, no changes are observed.

Compared with the results obtained with an iso-damping, the system stability in regard to the pair of variables $\left(\theta, \frac{\eta_{x}}{\eta_{y}}\right)$ is not straightforward. Whereas in the case of an iso-damping stability areas changes were similar in regard to adding damping whatever the values of $\theta$, in the case of a non-proportional damping, variation of the system stability is more complex, especially for planar friction (Figures 7(a) and (c)). For example, an increase of the damping ratio $\frac{\eta_{y}}{\eta_{x}}$ with $\eta_{y}$ constant decreases the stability for $\theta=[0 ;-54.4]^{\circ}$ but slightly increases the stability for $\theta=[-54.4 ;-90]^{\circ}$ (see Figure 7(a)). On the contrary, an increase of the ratio damping $\frac{\eta_{x}}{\eta_{y}}$ with $\eta_{x}$ constant has no noticeable effect on the stability for $\theta=[0 ;-45]^{\circ}$ whereas it decreases the stability for $\theta=[-45 ;-90]^{\circ}$ (see Figure $7(\mathrm{c})$ ). Thus, even if it is possible to increase the stability of the non proportional damped system in a specific sliding direction by modifying the damping ratio, this may decrease the stability of the system in another direction.

\section{[Figure 7 about here.]}

To explore and develop these initial observations on the role of non-proportional damping more deeply and to investigate the changes brought about by the additional damping contribution through planar friction, we propose to plot 
the variations of the real parts and frequencies for two specific values of $\theta\left(\theta=-30^{\circ}\right.$ and $\left.\theta=-60^{\circ}\right)$. The results are displayed in Figures 8. The variation of the eigenvalues in the complex plane are also plotted in Figure 9.

First of all, the classical results on the effect of non-proportional damping for mechanical system with rectilinear friction are found again[10,12,23]: if damping is spread non-equally over the two modes, shifting and smoothing effects can be seen on the variations of the real part and the coalescence curves: whereas the variation of the real part only occurs at the Hopf bifurcation point in the case of iso-damping $\eta_{y}=\eta_{x}$ (see Figure 6(d)), the real parts associated with stable and unstable modes increase and decrease, respectively, with the variation of the friction coefficient in the case of non-proportional damping $\eta_{y} \neq \eta_{x}$ (see Figure 8(e)). Then, if the smoothing effect prevails, added damping acts in an unintuitive way by destabilising the system, as illustrated in Figure 8(e): the system with non-proportional damping (i.e. $\frac{\eta_{y}}{\eta_{x}}=0.5$ and $\eta_{y}=0.02$ in Figure 8(e)) becomes unstable from a lower value of the friction coefficient than in the case of the system with iso-damping (i.e. $\frac{\eta_{y}}{\eta_{x}}=1$ and $\eta_{y}=0.02$ in Figure 8(e)) while damping $\eta_{x}$ increases (for the same value of $\eta_{y}$ ). As a consequence, increasing the gap in damping between the two modes tends to decrease the stability range as previously explained by Hoffmann and Gaul [21] and Fritz et al. [23]. Moreover, the merging between the two modes is imperfect in the case of non-proportional damping as illustrated in Figure 8(f): the difference between the frequencies of the stable and unstable modes depends on the structural damping ratio $\frac{\eta_{y}}{\eta_{x}}$. Table 4 gives the friction coefficients at the Hopf bifurcation point and the corresponding frequency of the unstable mode in all configurations. Figure 9 allows us to track the unstable mode on the coalescence patterns via the complex plane. Results reveal that the unstable mode is the least damped mode. Thus, the unstable mode may be the mode that initially has the highest or lowest frequency value depending on the ratio between the coefficients $\eta_{x}$ and $\eta_{y}$ (see [22, 23] for more details). In our case, when $\frac{\eta_{y}}{\eta_{x}}=0.5$ the unstable mode corresponds to the one with a frequency of $75 \mathrm{~Hz}$ for a friction coefficient equal to zero. When the ratio between $\eta_{y}$ and $\eta_{x}$ is reversed, the unstable mode is the mode with a frequency of $100 \mathrm{~Hz}$ for a coefficient of friction equal to zero.

We now discuss in details the case of the non-proportional damped system with planar friction and the notion of destabilisation paradox with the concept of lowering and smoothing effects in this case. Figures 8(a-d) and 9(a,b) display the real parts and frequencies for two specific values of $\theta\left(\theta=-30^{\circ}\right.$ and $\left.\theta=-60^{\circ}\right)$ and the corresponding variation of the eigenvalues in the complex plane, respectively. Table 4 gives the friction coefficient at the Hopf bifurcation point and the corresponding frequency of the unstable mode. Firstly, for a sliding velocity direction close to the x-axis (i.e. $\theta=-30^{\circ}$ ), an increase of the ratio $\frac{\eta_{y}}{\eta_{x}}$ (for a fixed value of $\eta_{y}=0.02$ ) generates a more unstable system (i.e. the Hopf bifurcation occurs at a lower value of the friction coefficient). This result is consistent with the destabilisation paradox for a system with rectilinear friction.

However, when $\theta=-60^{\circ}$ (which means that the sliding velocity direction is close to the y-axis), the Hopf bifurcation point is substantially independent of the ratio $\frac{\eta_{y}}{\eta_{x}}$. This result does not follow the trend of the classical notion of the destabilisation paradox found with rectilinear friction. The introduction of the additional damping $\tilde{\mathbf{C}}_{\mathbf{b}}$ due to planar friction alters the classical conclusions concerning the effect of the non-proportional damping. The changes on the system stability remain small but are not negligible if one is interested in making a mechanical system more stable through adding modal damping. The sole consideration of the modal damping ratio (which is a classical result for a mechanical system with rectilinear friction) is not enough to ensure a robust design versus the damping effect and the contribution of the 
damping induced by planar friction has to be taken into account.

We discuss now the mode coupling phenomena more specifically. For $\frac{\eta_{y}}{\eta_{x}}=1$, the imperfect merging (i.e. difference between frequencies of the stable and unstable modes), that can be seen in Figure 8(b) and (d), depends only on the additional damping $\tilde{\mathbf{C}}_{\mathbf{b}}$ due to planar friction. For all the other ratios (i.e. $\frac{\eta_{y}}{\eta_{x}} \neq 1$ ) this frequency gap is a combination of both the additional damping $\tilde{\mathbf{C}}_{\mathbf{b}}$ due to planar friction and the non-proportional damping $\frac{\eta_{y}}{\eta_{x}}$. Figures 9 (a) and (b) reveals an unexpected effect due to the presence of the additional damping $\tilde{\mathbf{C}}_{\mathbf{b}}$. As illustrated for two sliding velocity directions $\left(\theta=-30^{\circ}\right.$ and $\theta=-60^{\circ}$ in Figures 9(a) and (b) respectively), the unstable mode is not directly related to the ratio $\frac{\eta_{y}}{\eta_{x}}$ contrary to a classical result with rectilinear friction. In fact, the sliding direction seems to be the main factor determining which mode becomes unstable. For $\theta=-30^{\circ}$, the unstable mode corresponds to the mode with a frequency of $100 \mathrm{~Hz}$ for $\mu=0$ (the mode whose frequency is higher for $\mu=0$ ). On the contrary, for $\theta=-60^{\circ}$ the unstable mode is the mode with a frequency of $75 \mathrm{~Hz}$ for $\mu=0$ (the mode whose frequency is lower for $\mu=0$ ), even if the unstable mode frequency becomes larger than the stable mode frequency after the imperfect coalescence. Thus, it can be concluded that the additional damping contribution due to planar friction can drastically modify the coalescence patterns. The extension of the well-known destabilisation paradox due to the presence of non-proportional damping is not so intuitive and phenomena such as the lowering and smoothing effects are more complex. Considering planar friction, one of the key parameters that alters the conclusions obtained with rectilinear friction is the sliding velocity direction.

All these results show that the classical trends pointed out for a system with rectilinear friction cannot be extrapolated to a system with planar friction. Even if the smoothing effect is still observed (i.e. the fact that added damping may destabilise the system, as previously discussed and illustrated in Figure 8(a)), planar friction can either reinforce or compensate the destabilisation paradox (see for example the comparison between the two configurations shown in 8(a) and (c)). Thus, the effect of non-iso-damping for a system with planar friction is complex and the extension of the destabilisation paradox needs to be done in conjunction with the role of key parameters related to planar friction.

[Figure 8 about here.]

[Figure 9 about here.]

[Table 4 about here.]

\section{Conclusion}

In this paper the stability of a 3-D model with planar or rectilinear friction has been studied for different configurations by considering the influence of the equilibrium, the system's stiffness and the sliding direction.

In a first phase, the stability results for the undamped system with planar or rectilinear friction have shown significant differences in the stable and unstable areas. The critical friction coefficients at the Hopf bifurcation point are lower in the case of planar friction, which may be interpreted as a destabilisation due to the damping term introduced by the linearisation of the friction force direction. This damping leads to a smoothing of the bifurcations curves, i.e. a nonperfect mode coalescence. By increasing the sliding speed, the damping term is smaller and the complex eigenvalues corresponding to planar friction are close to those obtained with the rectilinear assumption, except in the neighbourhood 
of the Hopf bifurcation point. The effect of stiffness is similar for planar and rectilinear friction : the mode coupling effect is mainly affected by the proximity of the frequencies of the modes without friction. Compared with the rectilinear friction case, the sliding direction has a greater influence on the coalescence pattern: for some directions, a crossing of the two modes involved in the instability is observed.

In a second phase, the combined effect of the damping in the structure and the planar friction has been discussed. In terms of stability areas, the introduction of damping brings the results obtained with planar or rectilinear friction closer. However, this obviously depends on the ratio between the different damping terms. An extension of the destabilisation paradox due to the presence of damping and phenomena such as the lowering and smoothing effects has also been undertaken by considering the specific case of a system with planar friction. It was demonstrated that the additional damping contribution due to the consideration of planar friction can drastically modify the coalescence patterns and the associated instability phenomena are not so intuitive. In addition to the damping ratio and the friction coefficient, the sliding velocity direction appeared as one of the predominant factors.

\section{Acknowledgments}

The authors would like to thank the financial support provided by Celya (Centre Lyonnais d'Acoustique, Université de Lyon). J-J. Sinou acknowledges the support of the Institut Universitaire de France.

\section{A Calculation of Routh-Hurwitz coefficients}

In this section, the Routh-Hurwitz coefficients are calculated in the general case (for the system with planar friction and damping). The simplified expressions for the undamped system with planar friction and the system with rectilinear friction with damping are given.

First of all, after calculation the coefficients $a_{3}, a_{2}, a_{1}$ and $a_{0}$ of Equation 23 are given by

$$
\begin{gathered}
a_{3}=\frac{m c_{x}+m c_{y}+\mu m c_{b}}{m^{2}} \\
a_{2}=\frac{\mu\left(c_{x} c_{b} \cos ^{2} \theta+c_{y} c_{b} \sin ^{2} \theta-m k_{1} \tan \alpha \cos \theta-m k_{2} \tan \beta \sin \theta\right)+m k_{1}+m k_{2}+c_{x} c_{y}}{m^{2}} \\
a_{1}=\left(-\mu^{2}\left(c_{b} k_{1} \cos \theta \tan \alpha+c_{b} k_{2} \sin \theta \tan \beta\right)+\mu\left(c_{b} k_{2} \sin ^{2} \theta+c_{b} k_{1} \cos ^{2} \theta-c_{x} k_{2} \tan \beta \sin \theta\right.\right. \\
\left.\left.-c_{y} k_{1} \tan \alpha \cos \theta\right)+c_{x} k_{2}+c_{y} k_{1}\right) / m^{2} \\
a_{0}=\frac{-\mu\left(k_{1} k_{2} \tan \beta \sin \theta+k_{2} k_{1} \tan \alpha \cos \theta\right)+k_{1} k_{2}}{m^{2}}
\end{gathered}
$$

where $c_{b}=\frac{R_{n}^{e}}{V}$. 


\section{A.1 General case : planar friction with damping}

The coefficients of the Routh-Hurwitz criterion are now given in the general case :

$$
\begin{gathered}
a=a_{0}=\frac{-\mu\left(k_{1} k_{2} \tan \beta \sin \theta+k_{2} k_{1} \tan \alpha \cos \theta\right)+k_{1} k_{2}}{m^{2}} \\
b=a_{3}=\frac{m c_{x}+m c_{y}+\mu m c_{b}}{m^{2}} \\
c=a_{2} a_{3}-a_{1}=\left(\mu^{2}\left(c_{y} c_{b}^{2} \sin ^{2} \theta+c_{x} c_{b}^{2} \cos ^{2} \theta\right)\right. \\
+\mu\left(\left(\left(c_{y}^{2}+c_{x} c_{y}\right) c_{b}-c_{b} k_{2} m\right) \sin ^{2} \theta-c_{y} k_{2} m \tan \beta \sin \theta+\left(\left(c_{x} c_{y}+c_{x}^{2}\right) c_{b}-c_{b} k_{1} m\right) \cos ^{2} \theta\right. \\
\left.\left.-c_{x} k_{1} m \tan \alpha \cos \theta+\left(c_{b} k_{2}+c_{b} k_{1}\right) m+c_{x} c_{y} c_{b}\right)+\left(c_{y} k_{2}+c_{x} k_{1}\right) m+c_{x} c_{y}^{2}+c_{x}^{2} c_{y}\right) / m^{3} \\
d=a_{1}\left(a_{2} a_{3}-a_{1}\right)-a_{0} a_{3}^{2}=-\left(b_{4} \mu^{4}+b_{3} \mu^{3}+b_{2} \mu^{2}+b_{1} \mu+b_{0}\right) / m^{5}
\end{gathered}
$$

where $b_{4}, b_{3}, b_{2}, b_{1}$ and $b_{0}$ are defined by :

$$
\begin{aligned}
& b_{4}=c_{y} c_{b}^{3} k_{2} \tan \beta \sin ^{3} \theta+c_{y} c_{b}^{3} k_{1} \tan \alpha \cos \theta \sin ^{2} \theta+c_{x} c_{b}^{3} k_{2} \tan \beta \cos ^{2} \theta \sin \theta+c_{x} c_{b}^{3} k_{1} \tan \alpha \cos ^{3} \theta \\
& b_{3}=-c_{y} c_{b}^{3} k_{2} \sin ^{4} \theta+\left(\left(c_{y}+2 c_{x}\right) c_{y}-k_{2} m\right) c_{b}^{2} k_{2} \tan \beta \sin ^{3} \theta \\
& +\left(\left(-c_{x} k_{2}-c_{y} k_{1}\right) c_{b}^{3} \cos ^{2} \theta+\left(\left(2 c_{y}+c_{x}\right) c_{y}-k_{2} m\right) c_{b}^{2} k_{1} \tan \alpha \cos \theta\right. \\
& \left.-c_{y} c_{b} k_{2}^{2} m \tan ^{2} \beta\right) \sin ^{2} \theta+\left(\left(\left(c_{y}+2 c_{x}\right) c_{x}-k_{1} m\right) c_{b}^{2} k_{2} \tan \beta \cos ^{2} \theta\right. \\
& \left.+\left(-c_{y}-c_{x}\right) c_{b} k_{1} k_{2} m \tan \alpha \tan \beta \cos \theta+c_{b}^{2} k_{2}^{2} m \tan \beta+c_{x} c_{y} c_{b}^{2} k_{2} \tan \beta\right) \sin \theta \\
& -c_{x} c_{b}^{3} k_{1} \cos ^{4} \theta+\left(\left(2 c_{y}+c_{x}\right) c_{x}-k_{1} m\right) c_{b}^{2} k_{1} \tan \alpha \cos ^{3} \theta \\
& -c_{x} c_{b} k_{1}^{2} m \tan ^{2} \alpha \cos ^{2} \alpha+\left(k_{1} m+c_{x} c_{y}\right) c_{b}^{2} k_{1} \tan \alpha \cos \theta \\
& b_{2}=\left(k_{2} m+\left(-c_{y}^{2}-c_{x} c_{y}\right)\right) c_{b}^{2} k_{2} \sin ^{4} \theta+\left(\left(c_{y}-c_{x}\right) k_{2} m+\left(c_{x} c_{y}^{2}+c_{x}^{2} c_{y}\right)\right) c_{b} k_{2} \tan \beta \sin ^{3} \theta \\
& +\left(\left(2 k_{1} k_{2} m+\left(-c_{x} c_{y}-c_{x}^{2}\right) k_{2}+\left(-c_{y}^{2}-c_{x} c_{y}\right) k_{1}\right) c_{b}^{2} \cos ^{2} \theta+\left(\left(c_{x}-c_{y}\right) k_{2} m\right.\right. \\
& \left.\left.+\left(c_{y}+c_{x}\right) c_{y}^{2}\right) c_{b} k_{1} \tan \alpha \cos \theta+\left(\left(-c_{b}^{2}-c_{x} c_{y} \tan ^{2} \beta\right) k_{2}-c_{b}^{2} k_{1}\right) k_{2} m-2 c_{x} c_{y} c_{b}^{2} k_{2}-c_{y}^{2} c_{b}^{2} k_{1}\right) \sin ^{2} \theta \\
& +\left(\left(\left(c_{y}-c_{x}\right) k_{1} m+\left(c_{y}+c_{x}\right) c_{x}^{2}\right) c_{b} k_{2} \tan \beta \cos ^{2} \theta+\left(-c_{y}^{2}-c_{x}^{2}\right) k_{1} k_{2} m \tan \alpha \tan \beta \cos \theta\right. \\
& \left.+\left(\left(c_{y}-c_{x}\right) k_{2}-2 c_{y} k_{1}\right) c_{b} k_{2} m \tan \beta+\left(c_{y}+c_{x}\right) c_{x} c_{y} c_{b} k_{2} \tan \beta\right) \sin \theta+\left(k_{1} m+\left(-c_{y}-c_{x}\right) c_{x}\right) c_{b}^{2} k_{1} \cos ^{4} \theta \\
& +\left(\left(c_{x}-c_{y}\right) k_{1} m+\left(c_{y}+c_{x}\right) c_{x} c_{y}\right) c_{b} k_{1} \tan \alpha \cos ^{3} \theta+\left(\left(\left(-c_{b}^{2}-c_{x} c_{y} \tan ^{2} \alpha\right) k_{1}-c_{b}^{2} k_{2}\right) k_{1} m\right. \\
& \left.-c_{x}^{2} c_{b}^{2} k_{2}-2 c_{x} c_{y} c_{b}^{2} k_{1}\right) \cos ^{2} \theta+\left(\left(\left(c_{y}+c_{x}\right) k_{1}-2 c_{x} k_{2}\right) m+\left(2 c_{y}+c_{x}\right) c_{x} c_{y}\right) c_{b} k_{1} \tan \alpha \cos \theta+c_{b}^{2} k_{1} k_{2} m
\end{aligned}
$$




$$
\begin{aligned}
b_{1}= & \left(\left(\left(c_{x}-c_{y}\right) k_{2}+\left(c_{y}-c_{x}\right) k_{1}\right) c_{b} k_{2} m+\left(-2 c_{y}-2 c_{x}\right) c_{x} c_{y} c_{b} k_{2}+\left(-c_{y}-c_{x}\right) c_{y}^{2} c_{b} k_{1}\right) \sin ^{2} \theta \\
& +\left(\left(2 k_{2}-2 k_{1}\right) m+\left(c_{x} c_{y}+c_{x}^{2}\right)\right) c_{x} c_{y} k_{2} \tan \beta \sin \theta+\left(\left(\left(c_{x}-c_{y}\right) k_{2}+\left(c_{y}-c_{x}\right) k_{1}\right) c_{b} k_{1} m\right. \\
& \left.+\left(-c_{y}-c_{x}\right) c_{x}^{2} c_{b} k_{2}+\left(-2 c_{y}-2 c_{x}\right) c_{x} c_{y} c_{b} k_{1}\right) \cos ^{2} \theta+\left(\left(2 k_{1}-2 k_{2}\right) m+\left(c_{y}^{2}+c_{x} c_{y}\right)\right) c_{x} c_{y} k_{1} \tan \alpha \cos \theta \\
& +\left(-c_{x} k_{2}^{2}+\left(c_{y}+c_{x}\right) k_{1} k_{2}-c_{y} k_{1}^{2}\right) c_{b} m-c_{x}^{2} c_{y} c_{b} k_{2}-c_{x} c_{y}^{2} c_{b} k_{1}
\end{aligned}
$$

$$
b_{0}=-\left(k_{2}-k_{1}\right)^{2} c_{x} c_{y} m+\left(-c_{x} c_{y}-c_{x}^{2}\right) c_{x} c_{y} k_{2}+\left(-c_{y}^{2}-c_{x} c_{y}\right) c_{x} c_{y} k_{1}
$$

In the general case, if the coefficients $a, b$ and $c$ are strictly positive, the value of the friction coefficient at the Hopf bifurcation $\mu_{c}$ such that $d\left(\mu_{c}\right)=0$ is a solution of a polynomial equation of degree 4 . This yields to more than one solution with a positive value of the friction coefficient which means that the mechanical system can be stable again after being destabilised by a mode coupling instability.

\section{A.2 Planar friction without damping}

The case of a system with planar friction and without damping $\left(c_{x}=c_{y}=0\right)$ is now studied. The Routh-Hurwitz coefficients are :

$$
\begin{gathered}
a=\frac{-\mu\left(k_{1} k_{2} \tan \beta \sin \theta+k_{2} k_{1} \tan \alpha \cos \theta\right)+k_{1} k_{2}}{m^{2}} \\
b=\frac{\mu m c_{b}}{m^{2}} \\
c=\frac{\mu c_{b}\left(k_{2} \cos ^{2} \theta+k_{1} \sin ^{2} \theta\right)}{m^{2}} \\
d=\frac{\mu^{2} c_{b}^{2}\left(k_{1}-k_{2}\right) \cos \theta \sin \theta}{m^{4}}\left(\left(k_{1}-k_{2}\right) \cos \theta \sin \theta-\mu\left(k_{1} \tan \alpha \sin \theta-k_{2} \tan \beta \cos \theta\right)\right)
\end{gathered}
$$

In this case the value of the friction coefficient at the Hopf bifurcation $\mu_{c}$ such that $d\left(\mu_{c}\right)=0$ is the solution of a polynomial equation of degree 1 and does not depend on $c_{b}$. There is only one value of $\mu_{c}$ for each sliding direction $\theta$, which means that contrary to the system with damping, the system without damping remains unstable when it is destabilised by a mode coupling instability.

\section{A.3 Rectilinear friction with damping}

The case of a system with rectilinear friction and damping $\left(c_{b}=0\right)$ is now studied. The Routh-Hurwitz coefficients are :

$$
\begin{gathered}
a=\frac{-\mu\left(k_{1} k_{2} \tan \beta \sin \theta+k_{2} k_{1} \tan \alpha \cos \theta\right)+k_{1} k_{2}}{m^{2}} \\
b=\frac{m c_{x}+m c_{y}}{m^{2}} \\
c=\frac{\mu\left(-k_{2} c_{y} \tan \beta \sin \theta-k_{1} c_{x} \tan \alpha \cos \theta\right) m+\left(c_{y} k_{2}+c_{x} k_{1}\right) m+c_{x} c_{y}\left(c_{x}+c_{y}\right)}{m^{3}} \\
d=\frac{\mu^{2} b_{2}+\mu b_{1}+b_{0}}{m^{5}}
\end{gathered}
$$

where

$$
b_{2}=\left(k_{2}^{2} \tan ^{2} \beta \sin ^{2} \theta+k_{1}^{2} \tan ^{2} \alpha \cos ^{2} \theta\right) c_{x} c_{y} m+\left(c_{y}^{2}+c_{x}^{2}\right) k_{1} k_{2} m \tan \alpha \tan \beta \sin \theta \cos \theta
$$




$$
\begin{gathered}
b_{1}=-c_{x} c_{y}\left(\left(\left(k_{2}-k_{1}\right) 2 m+\left(c_{x} c_{y}+c_{x}^{2}\right)\right) k_{2} \tan \beta \sin \theta+\left(\left(k_{1}-k_{2}\right) 2 m+\left(c_{y}^{2}+c_{x} c_{y}\right)\right) k_{1} \tan \alpha \cos \theta\right) \\
b_{0}=c_{x} c_{y}\left(\left(k_{1}-k_{2}\right)^{2} m+\left(c_{x} c_{y}+c_{x}^{2}\right) k_{2}+\left(c_{y}^{2}=c_{x} c_{y}\right) k_{1}\right)
\end{gathered}
$$

In this case the value of the friction coefficient at the Hopf bifurcation $\mu_{c}$ is the solution of a polynomial equation of degree 2. If the two solutions are real positive, the system can be stabilised again after being destabilised by a mode coupling instability.

\section{A.4 Rectilinear friction without damping}

In the case of a system with a rectilinear friction and without damping, the system stability has to be studied by calculating the eigenvalues instead of using the Routh-Hurwitz criterion. Details of this method can be found by instance in [13]. The friction coefficient at the Hopf bifurcation $\mu_{c}$ is solution of a polynomial equation of degree 2. Thus, the system can be stabilised again after being previously destabilised by a mode coupling instability.

\section{References}

[1] D.A. Crolla and Lang A.M. Brake noise and vibration - state of art. Vehicule Tribology, Tribology Series, 18:165174, 1991.

[2] N.M. Kindkaid, O.M. O'Reilly, and P. Papadopoulos. Automative disc brake squeal. Journal of Sound and Vibration, 267:105-166, 2003.

[3] H. Ouyang, W. Nack, Y. Yuan, and F. Chen. Numerical analysis of automative disc brake squeal : a review. International Journal of Vehicle Noise and Vibration, 1:207-231, 2005.

[4] R.A. Ibrahim. Friction-induced vibration, chatter, squeal and chaos part ii : Dynamics and modelling. Applied Mechanics Review, 47:227-253, 1994.

[5] J.T. Oden and J.A.C. Martins. Models and computational methods for dynamic friction phenomena. Computer Methods in Applied Mechanics and Engineering, 52:527-634, 1987.

[6] A. Akay. Acoustics of friction. Journal of the Acoustical Society of America, 111:1252-1548, 2002.

[7] R.A. Ibrahim. Friction-induced vibration, chatter, squeal and chaos part 1: mechanics of contact and friction. Applied Mechanics Review, 47:209-226, 1994.

[8] F. Massi, Y. Berthier, and L. Baillet. Contact surface topography and system dynamics of brake squeal. Wear, 265(11-12):1784-1792, 2008.

[9] R. Spurr. A theory of brake squeal. Proc. Auto. Div. Inst. Mech. Eng., 1:33-40, 1961.

[10] N. Hoffmann, M. Fischer, R. Allgaier, and L. Gaul. A minimal models for studying properties of the mode-coupling type instability in friction induced oscillations. Mechanics Research Communications, 29:197-205, 2002. 
[11] U. Von Wagner, P. Hagedorn, and D. Hochlenert. Minimal models for disk brake squeal. Journal of Sound and Vibration, 302:527-539, 2007.

[12] J-J. Sinou and L. Jézéquel. Mode coupling instability in friction-induced vibrations and its dependency on system parameters including damping. European Journal of Mechanics A/Solids, 26:106-122, 2007.

[13] F. Moirot. Étude de la stabilité d'un équilibre en présence de frottement de Coulomb. PhD thesis, École Polytechnique, 1998.

[14] F. Moirot and Q-S. Nguyen. Friction and Instabilities, chapter Some examples of friction-induced vibrations and instabilities, pages 137-178. Springer Vienna, Vienna, 2002.

[15] X. Lorang. Instabilité vibratoire des structures en contact frottant : Application au crissement des freins de TGV. $\mathrm{PhD}$ thesis, École Polytechnique, 2004.

[16] X. Lorang, F. Foy-Margiocchi, Q.S. Nguyen, and P.E. Gautier. Tgv disc brake squeal. Journal of Sound and Vibration, 293(3 - 5):735 - 746, 2006.

[17] D. Brizard, O. Chiello, J-J. Sinou, and X. Lorang. Performances of some reduced bases for the stability analysis of a disc/pads system in sliding contact. Journal of Sound and Vibration, 330:703-720, 2011.

[18] N.M. Kinkaid, O.M. O'Reilly, and P. Papadopoulos. On the transient dynamics of a multi-degree-of-freedom friction oscillator: a new mechanism for disc brake noise. Journal of Sound and Vibration, 287:901 - 917, 2005.

[19] S. Oberst and J.C.S. Lai. Nonlinear friction coupling in disc brake squeal. 18th international Congress on Sound and Vibration, Rio De Janeiro, Brazil, 10-14 July, pages 1- 8, 2011.

[20] Z Zhang, S. Oberst, and J.C.S. Lai. Instability analysis of friction oscillators with uncertainty in the friction law distribution. Proceedings of the Institution of Mechanical Engineers Part C: Journal of Mechanical Engineering Science, pages $1-11,2015$.

[21] N. Hoffmann and L. Gaul. Effects of damping on mode-coupling instability in friction induced oscillations. ZAMM Zeitschrift fur Angewandte Mathematik und Mechanik, 83(8):524-534, 2003.

[22] J-J. Sinou, G. Fritz, and L. Jézéquel. The role of damping and definition of the robust damping factor (rd-factor) for a self-exciting mechanism with constant friction. Journal of Vibration and Acoustics, 129(3):297-306, 2007.

[23] G. Fritz, J-J. Sinou, J-M. Duffal, and L. Jézéquel. Investigation of the relationship between damping and mode coupling patterns in case of brake squeal. Journal of Sound and Vibration, 307(3-5):591-609, 2007.

[24] O. N. Kirillov. Stabilizing and destabilizing perturbations of pt-symmetric indefinitely damped systems. Philosophical Transactions of the Royal Society A, 371:20120051, 2013.

[25] O.N. Kirillov. Destabilization paradox due to breaking the hamiltonian and reversible symmetry. International Journal of Non-linear Mechanics, 42(1):71-87, 2007. 
[26] O.N. Kirillov and A.O. Seyranian. The effect of small internal and external damping on the stability of distributed non-conservative systems. Journal of Applied Mathematics and Mechanics, 69(4):529-552, 2005.

[27] O.N. Kirillov and A.P. Seyranian. Stabilization and destabilization of a circulatory system by small velocitydependent forces. Journal of Sound and Vibration, 283:781-800, 2005.

[28] F. Massi and O. Giannini. Effect of damping on the propensity of squeal instability: An experimental investigation. The Journal of the Acoustical Society of America, 123(4):2017-2023, 2008.

[29] B. Hervé, J-J. Sinou, H. Mahé, and L. Jézéquel. Extension of the destabilization paradox to limit cycle amplitudes for a nonlinear self-excited system subject to gyroscopic and circulatory actions. Journal of Sound and Vibration, 323(3):944-973, 2009.

[30] B. Hervé, J-J. Sinou, H. Mahé, and L. Jézéquel. Analysis of squeal noise and mode coupling instabilities including damping and gyroscopic effects. European Journal of Mechanics A/Solids, 27(2):141-160, 2008.

[31] G. Meinsma. Elemantary proof of the routh-hurwitz test. Systems and Control Letter 25, (4):237-242, 1995.

[32] O. N. Kirillov. Non conservative stability problems of modern physics. De Gruyter, Berlin, Boston, $371,2013$. 


\section{List of Figures}

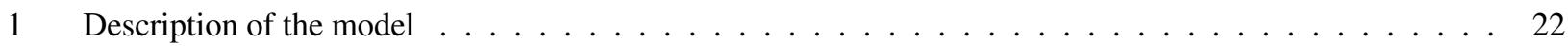

2 Stability area using the reference parameters . . . . . . . . . . . . . . . . . 23

3 Influence of stiffness difference $k_{1}-k_{2}$ with $k_{1}+k_{2}$ constant on (a-b) the stability area, (c-d) the real parts for $\theta=-30^{\circ}$, (e-f) and the frequencies for $\theta=-30^{\circ}$, for $(\mathrm{a}, \mathrm{c}, \mathrm{e})$ planar friction and $(\mathrm{b}, \mathrm{d}, \mathrm{f})$ rectilinear friction . . . . . . . . . . . . . . . . . . . . . . . . . . 24

4 Influence of sliding velocity on (a) the real parts and (b) the frequencies for $\theta=-30^{\circ} \ldots \ldots$

5 Influence of sliding velocity direction on (a) the real parts, (b) the frequencies and (c) the complex plane for a planar friction . . . . . . . . . . . . . . . . . . . . . . . 26

6 Influence of iso-damping on $(\mathrm{a}, \mathrm{b})$ the stability area, $(\mathrm{c}, \mathrm{d})$ the real parts for $\theta=-30^{\circ}$ and $(\mathrm{e}, \mathrm{f})$ the frequencies for $\theta=-30^{\circ}$ in case of $(\mathrm{a}, \mathrm{c}, \mathrm{e})$ planar friction or $(\mathrm{b}, \mathrm{d}, \mathrm{f})$ rectilinear friction . . . . . . . . . . . . 27

7 Influence of non-iso-damping with $(\mathrm{a}, \mathrm{b}) \eta_{y}$ constant or $(\mathrm{c}, \mathrm{d}) \eta_{x}$ constant on the stability area for $(\mathrm{a}, \mathrm{c})$ planar friction or $(\mathrm{b}, \mathrm{d})$ rectilinear friction . . . . . . . . . . . . . . . . . . . . 28

8 Influence of non-iso damping on (a,c,e) the real parts and (b,d,f) the frequencies with $\eta_{y}$ constant for (a,b) planar friction and $\theta=-30^{\circ},(\mathrm{c}, \mathrm{d})$ planar friction and $-60^{\circ},(\mathrm{e}, \mathrm{f})$ rectilinear friction and $\theta=-30^{\circ} \quad \ldots \quad$. 29

9 Influence of non-iso damping on the complex planes with $\eta_{y}$ constant for (a) planar friction and $\theta=-30^{\circ}$, (b) planar friction and $-60^{\circ}$ and $\left(\right.$ c) rectilinear friction and $\theta=-30^{\circ} \ldots \ldots \ldots$ 


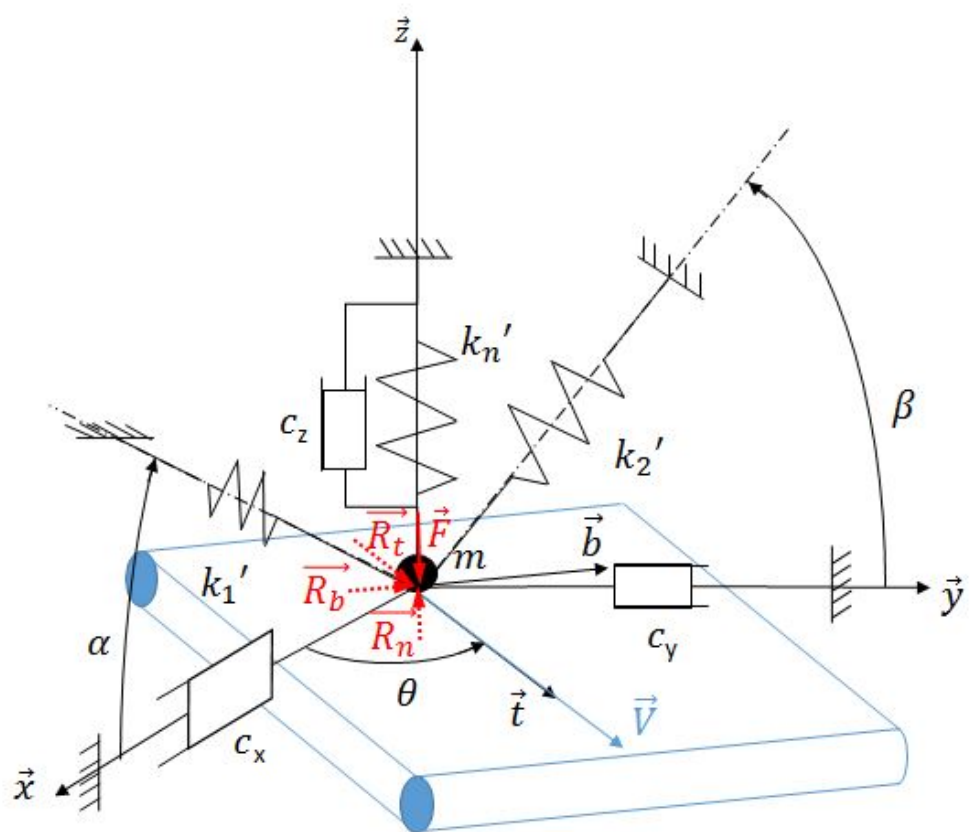

Figure 1: Description of the model 


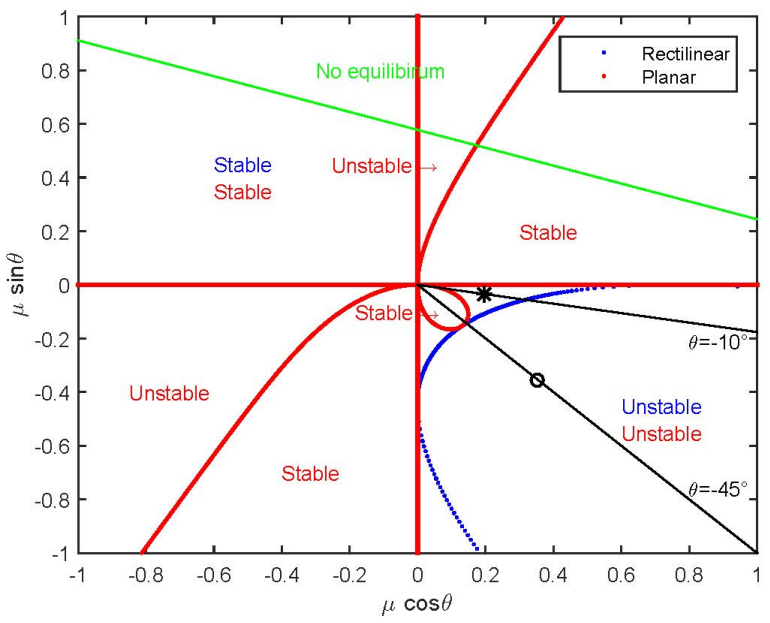

Figure 2: Stability area using the reference parameters 


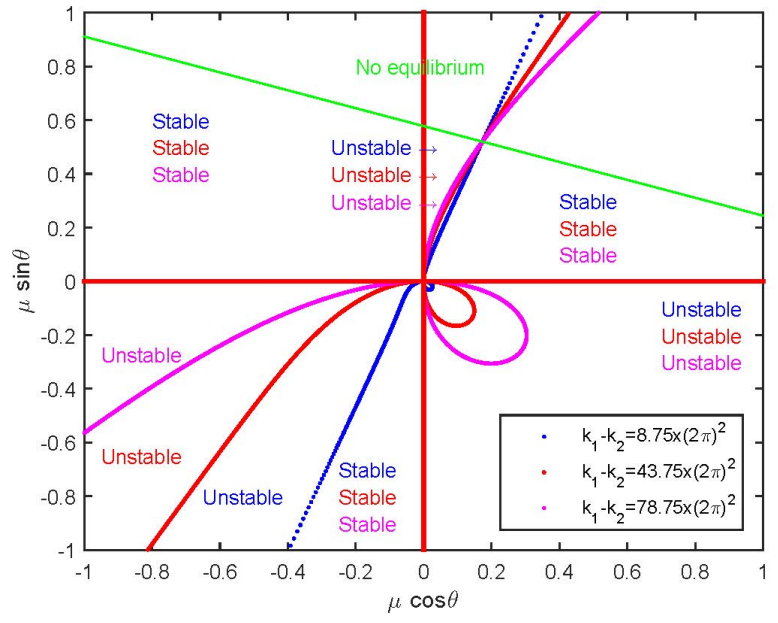

(a)

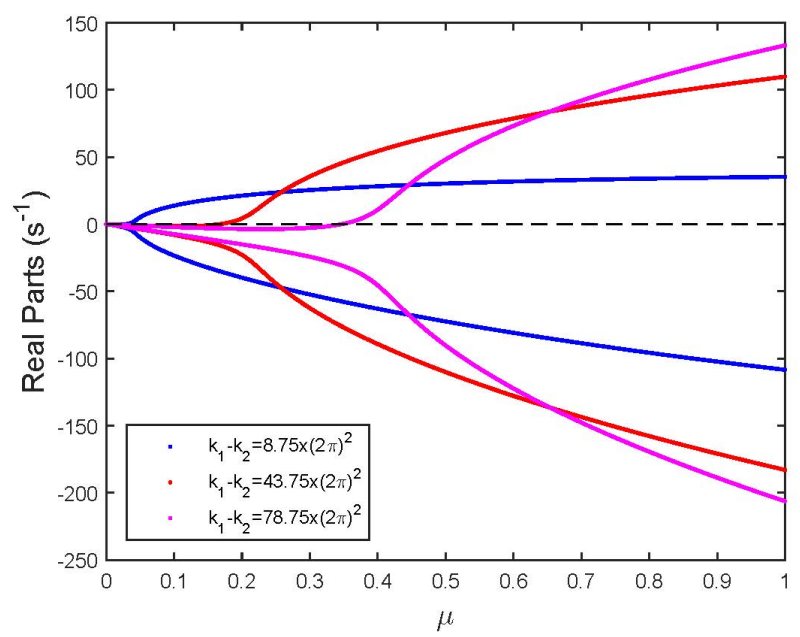

(c)

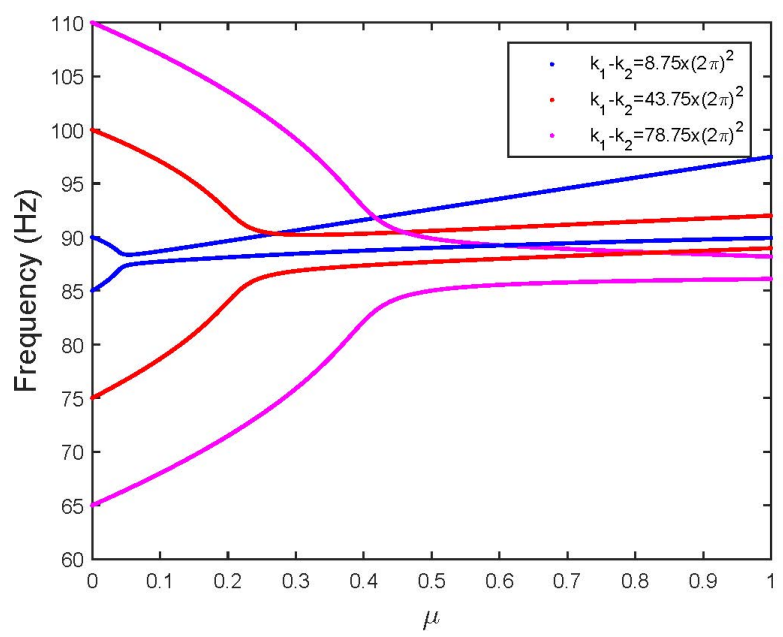

(e)

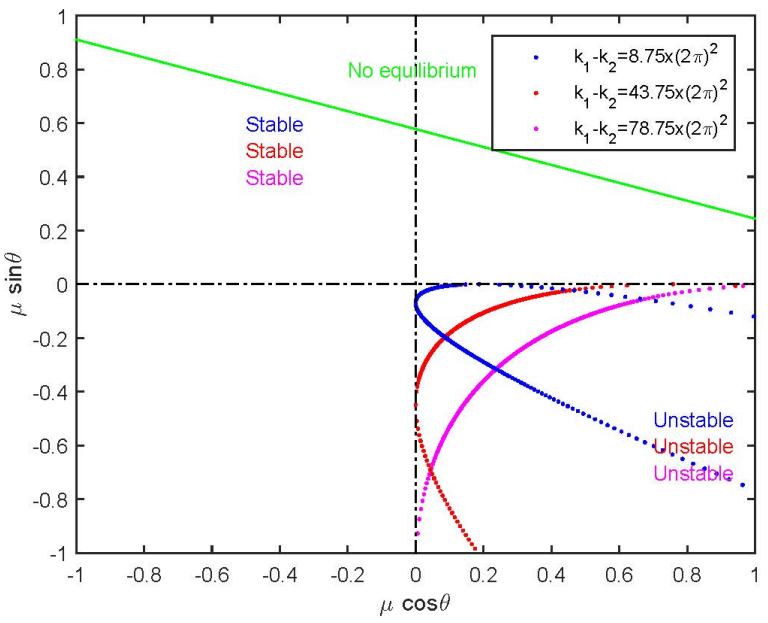

(b)

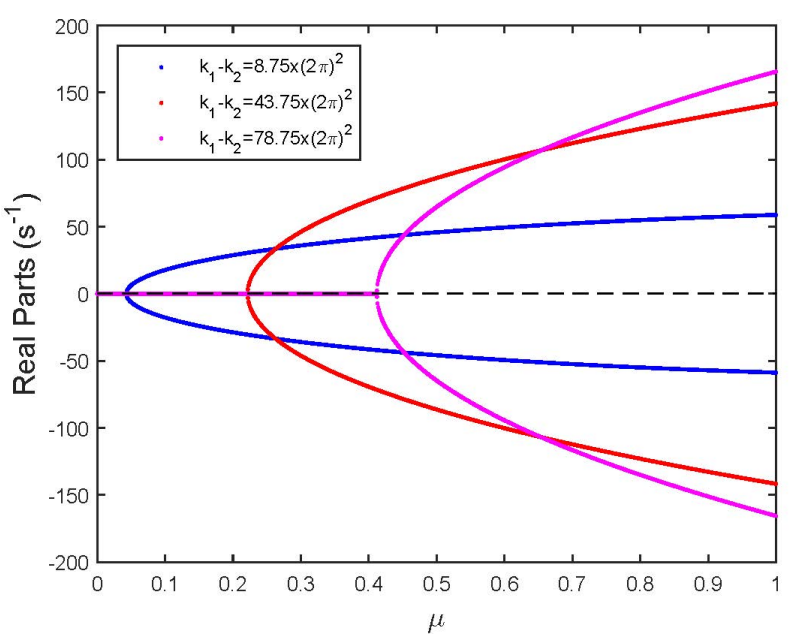

(d)

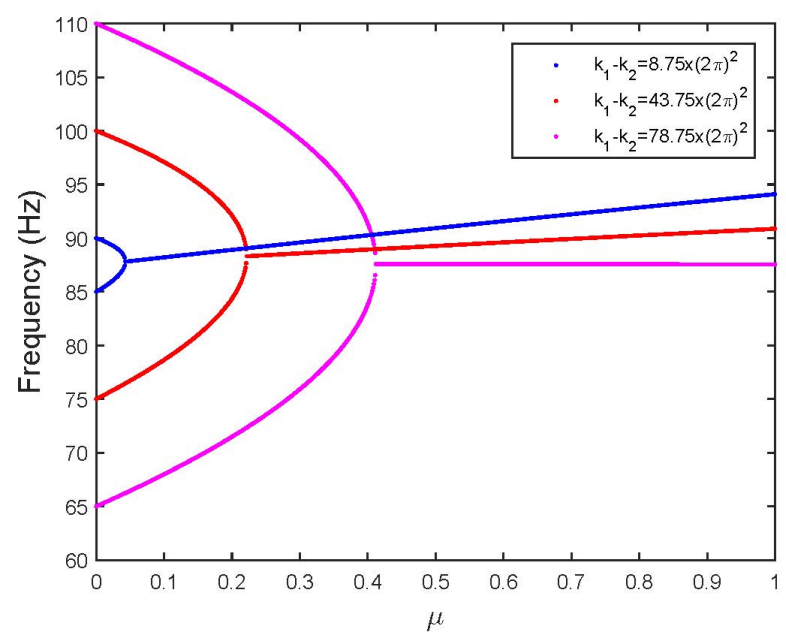

(f)

Figure 3: Influence of stiffness difference $k_{1}-k_{2}$ with $k_{1}+k_{2}$ constant on (a-b) the stability area, (c-d) the real parts for $\theta=-30^{\circ}$, (e-f) and the frequencies for $\theta=-30^{\circ}$, for $(\mathrm{a}, \mathrm{c}, \mathrm{e})$ planar friction and $(\mathrm{b}, \mathrm{d}, \mathrm{f})$ rectilinear friction 


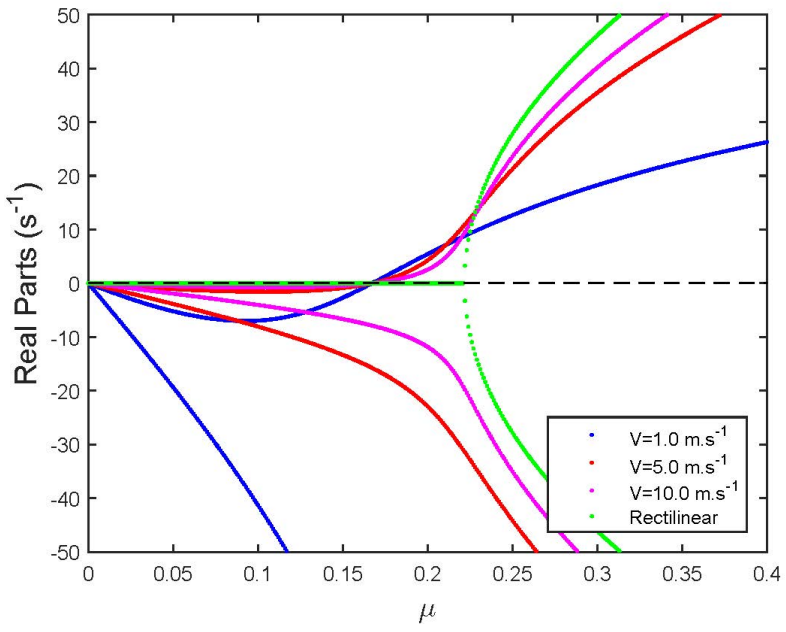

(a)

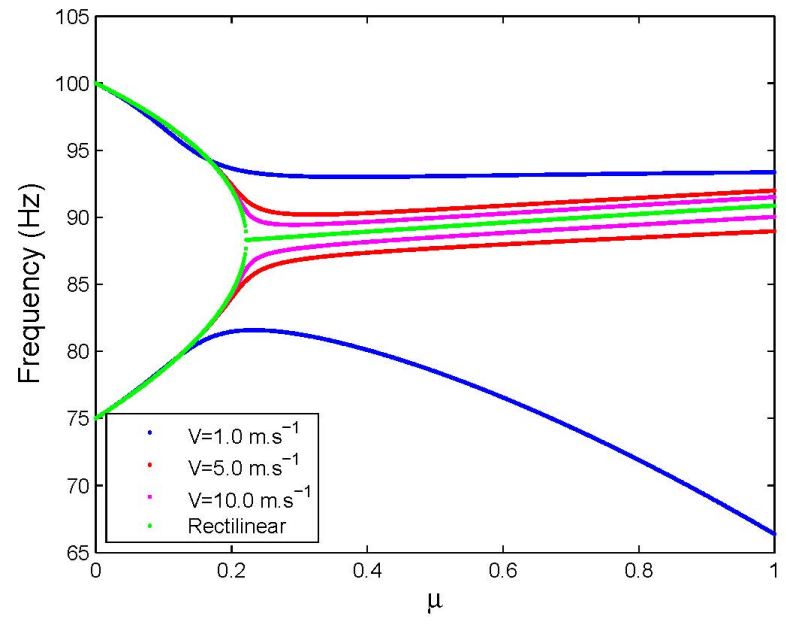

(b)

Figure 4: Influence of sliding velocity on (a) the real parts and (b) the frequencies for $\theta=-30^{\circ}$ 


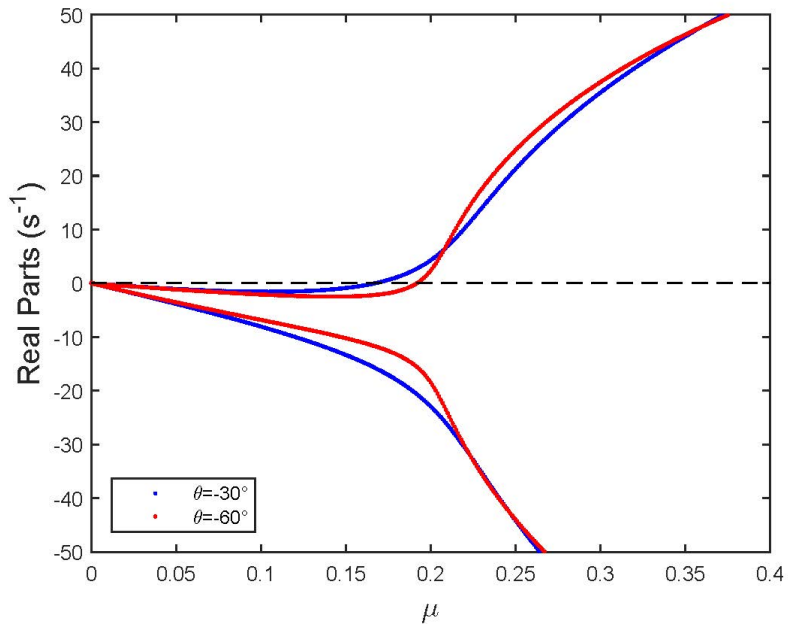

(a)

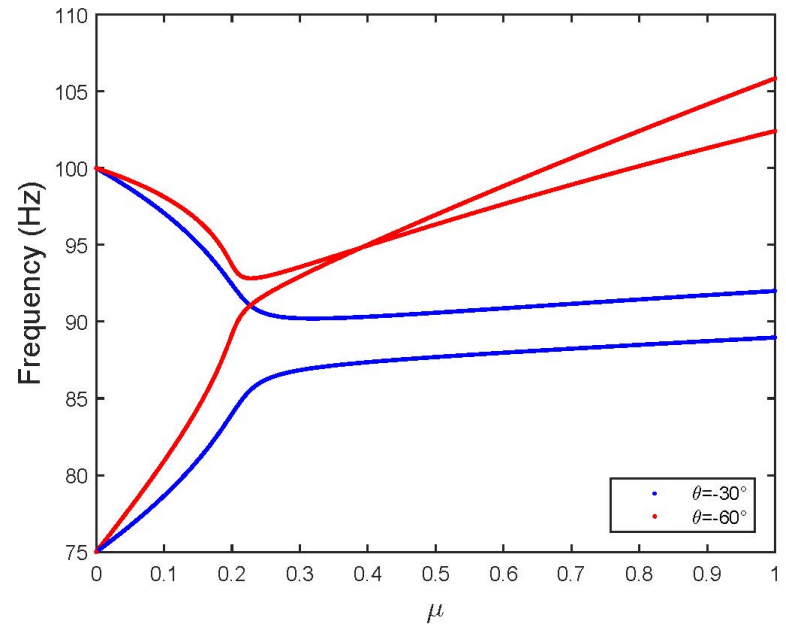

(b)

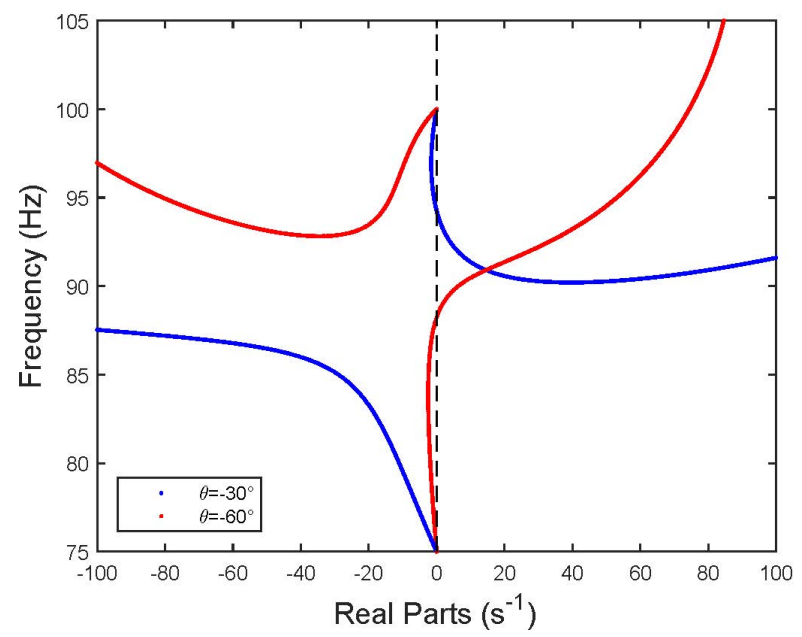

(c)

Figure 5: Influence of sliding velocity direction on (a) the real parts, (b) the frequencies and (c) the complex plane for a planar friction 


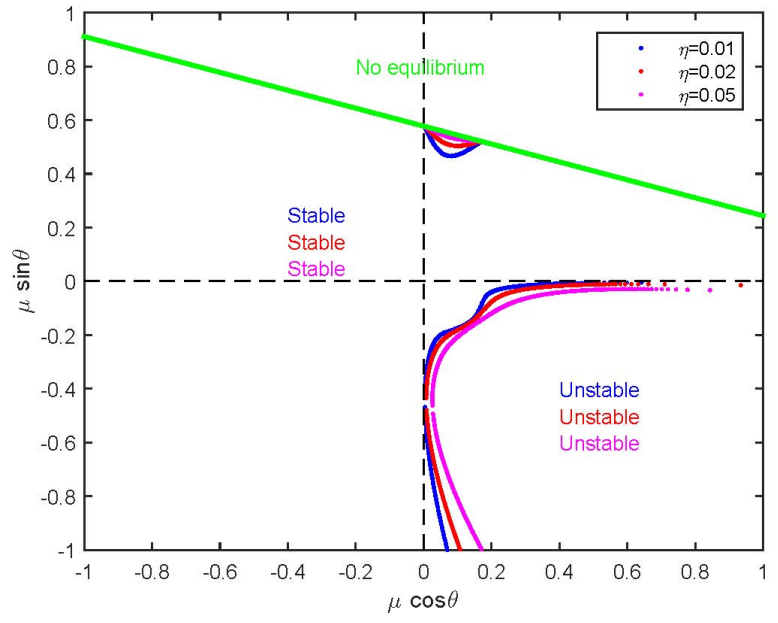

(a)

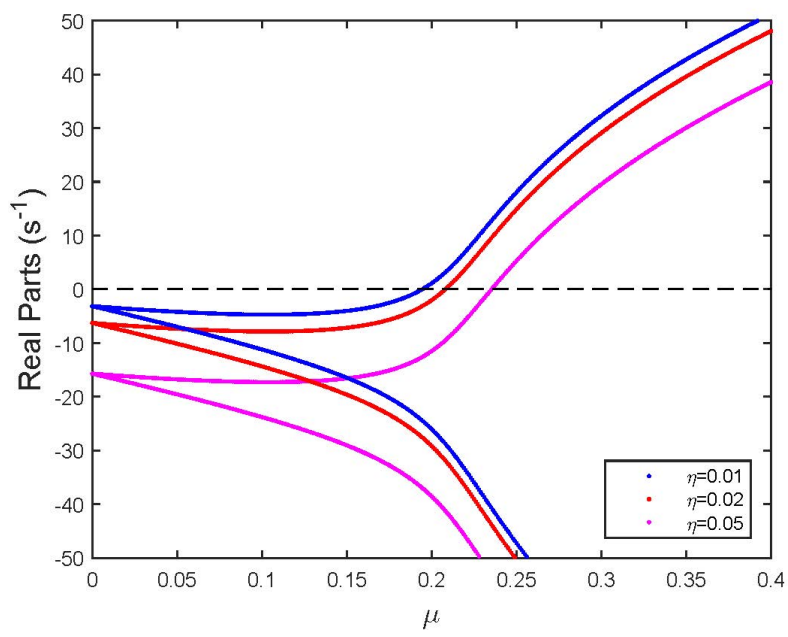

(c)

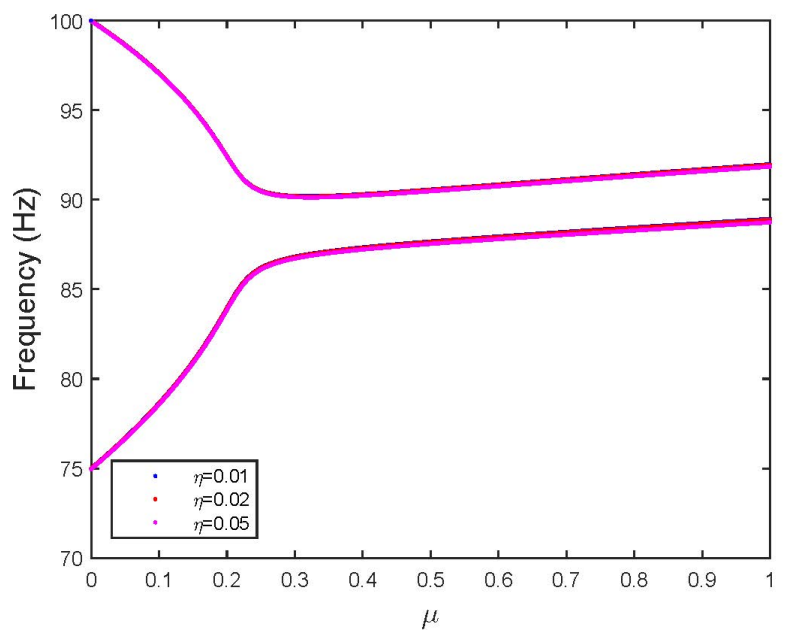

(e)

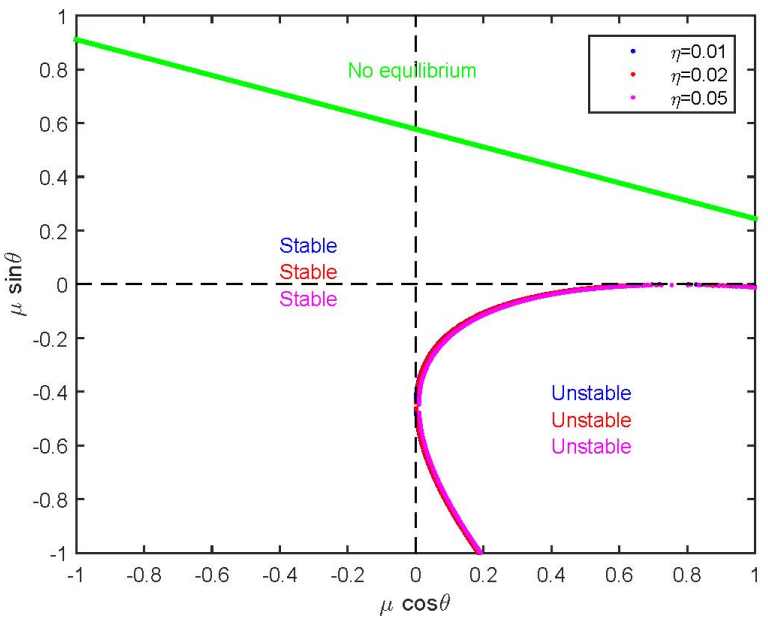

(b)

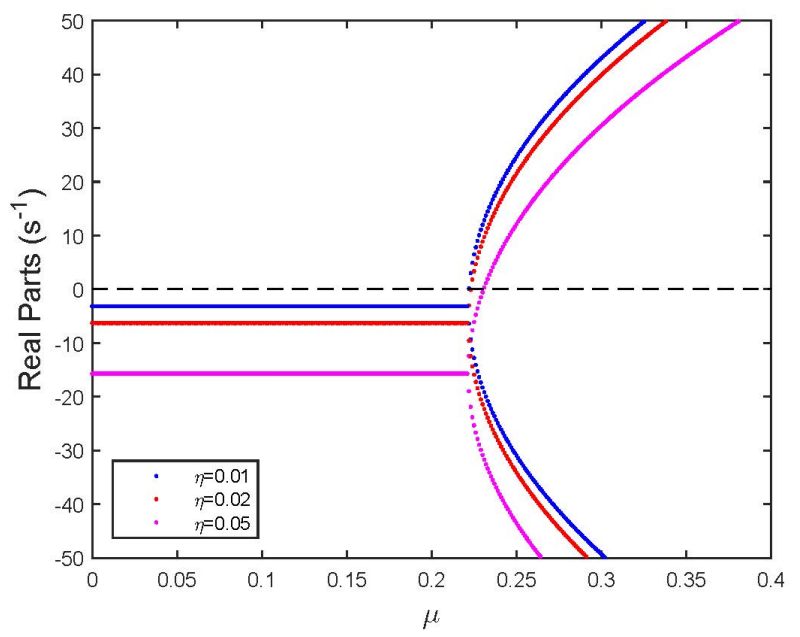

(d)

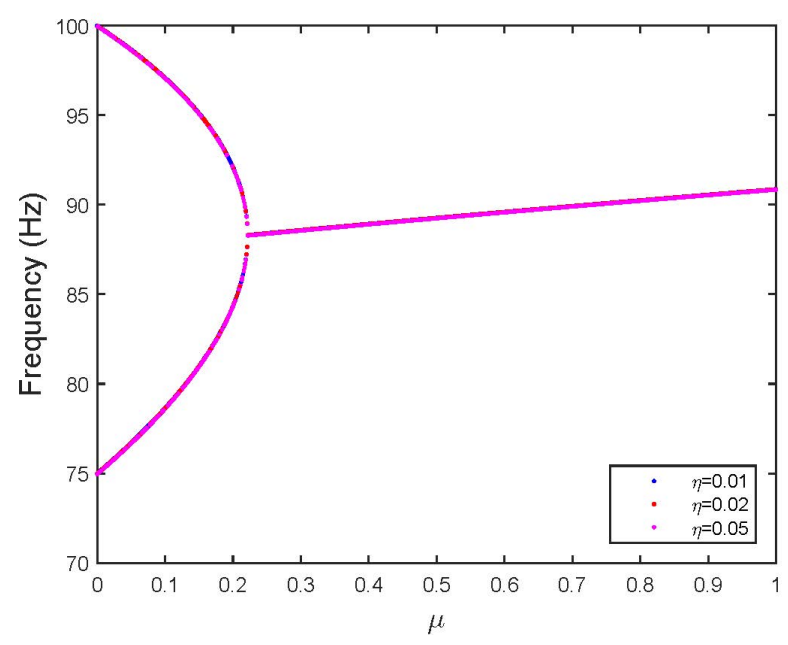

(f)

Figure 6: Influence of iso-damping on $(\mathrm{a}, \mathrm{b})$ the stability area, $(\mathrm{c}, \mathrm{d})$ the real parts for $\theta=-30^{\circ}$ and $(\mathrm{e}, \mathrm{f})$ the frequencies for $\theta=-30^{\circ}$ in case of $(\mathrm{a}, \mathrm{c}, \mathrm{e})$ planar friction or $(\mathrm{b}, \mathrm{d}, \mathrm{f})$ rectilinear friction 


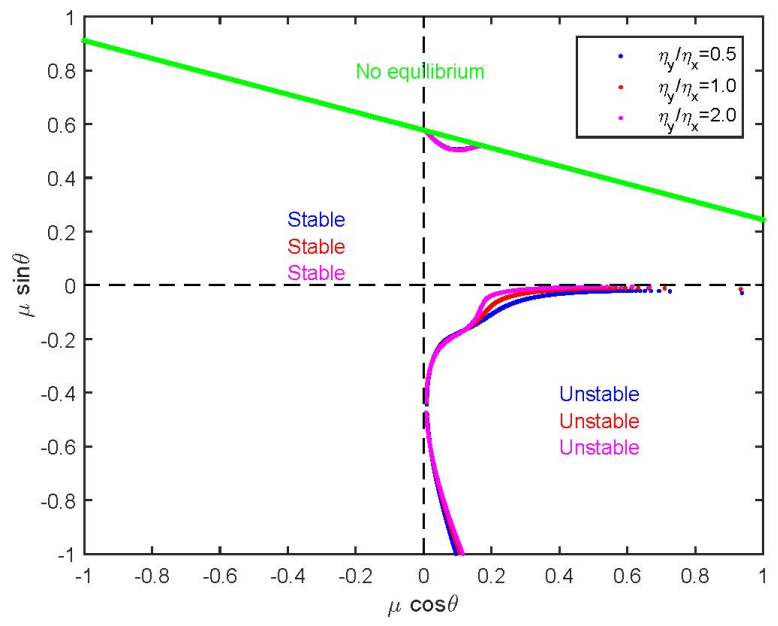

(a)

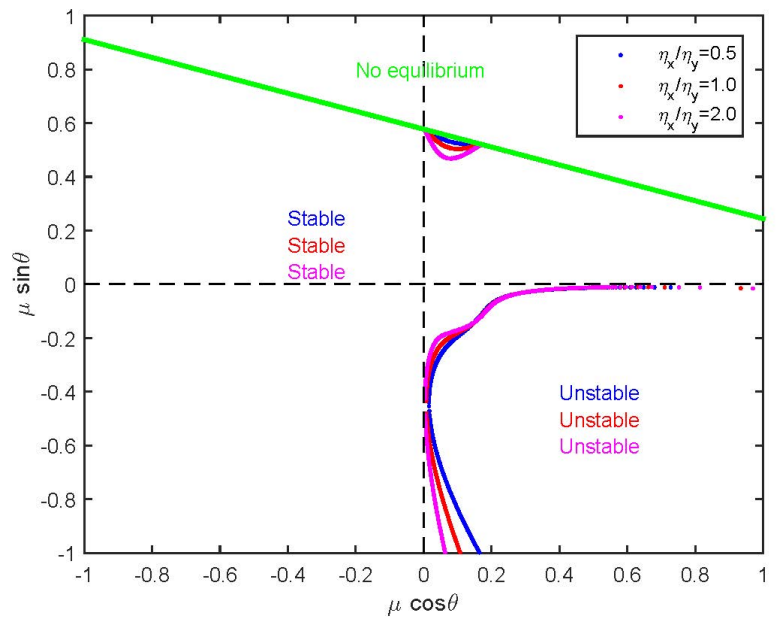

(c)

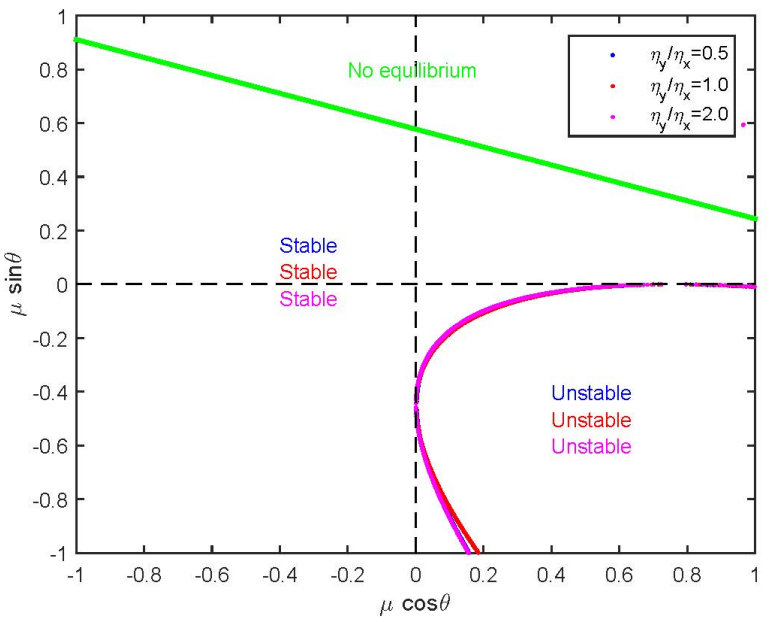

(b)

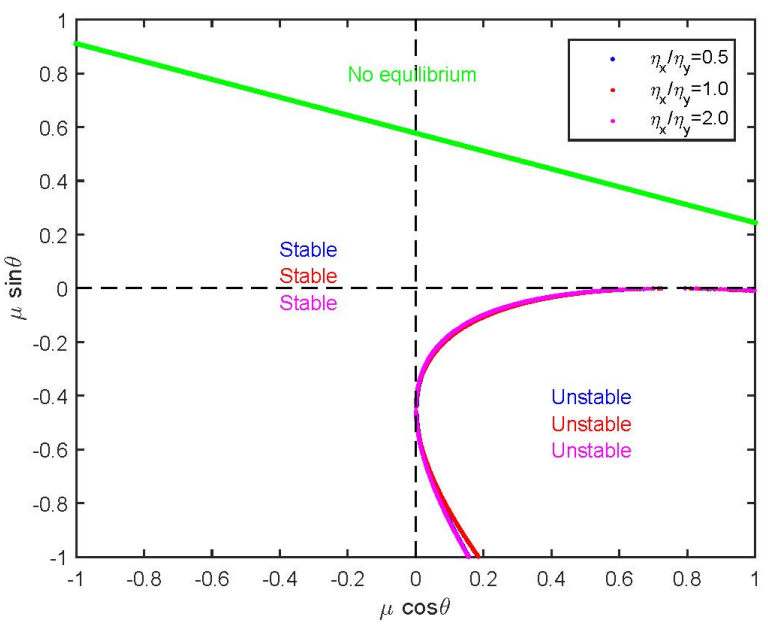

(d)

Figure 7: Influence of non-iso-damping with (a,b) $\eta_{y}$ constant or (c,d) $\eta_{x}$ constant on the stability area for (a,c) planar friction or $(b, d)$ rectilinear friction 


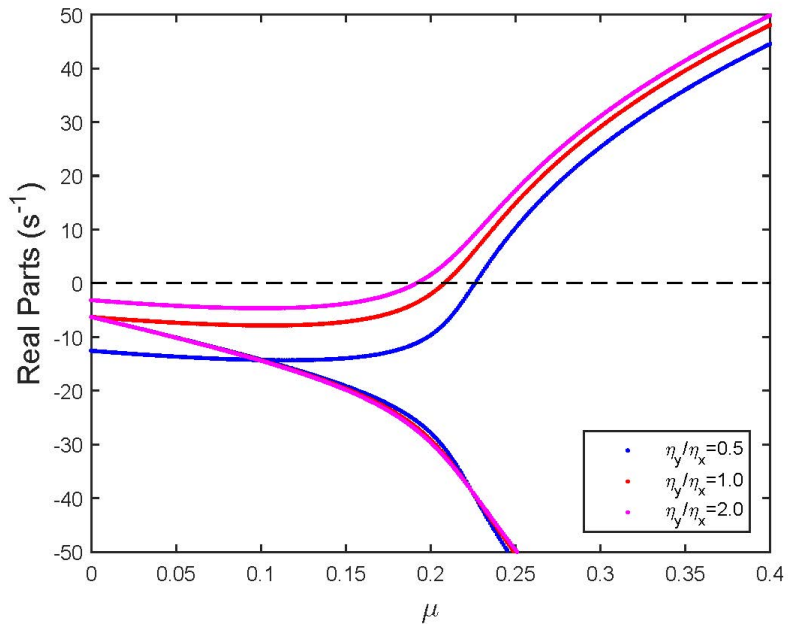

(a)

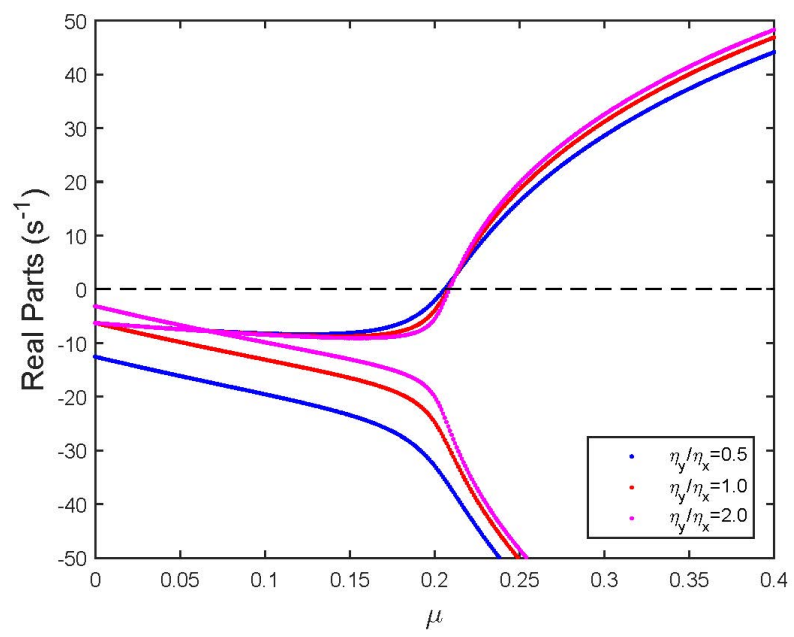

(c)

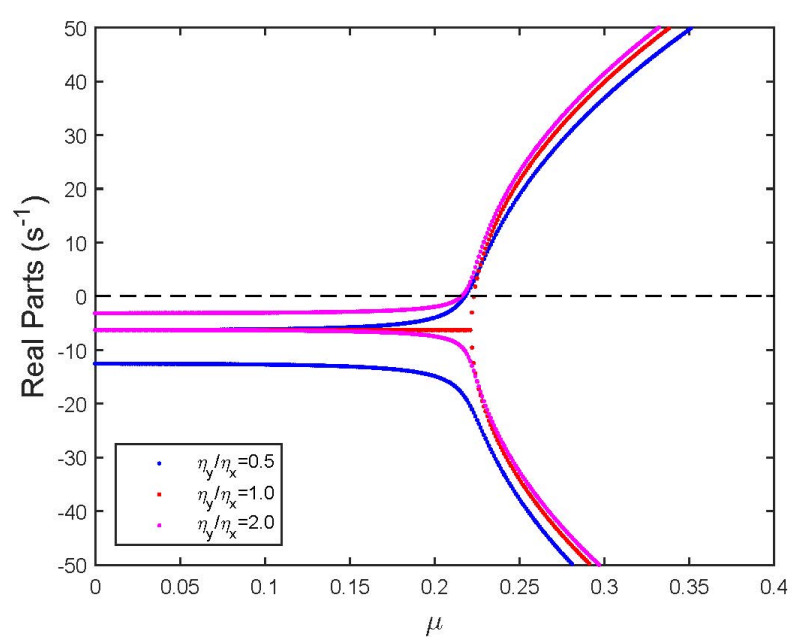

(e)

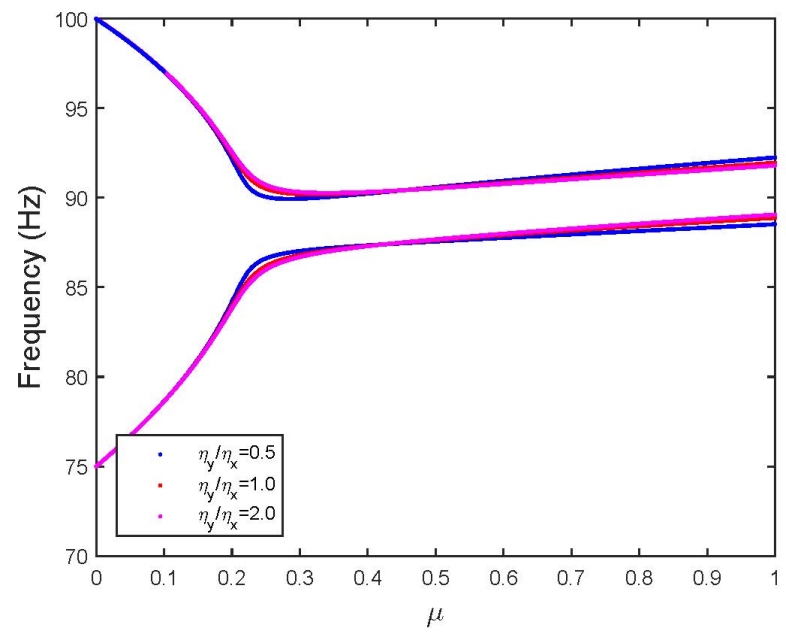

(b)

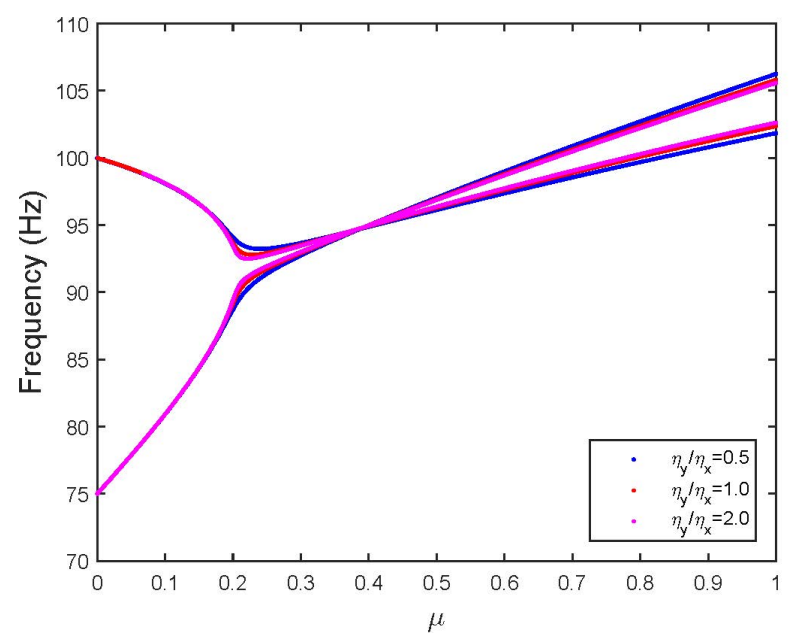

(d)

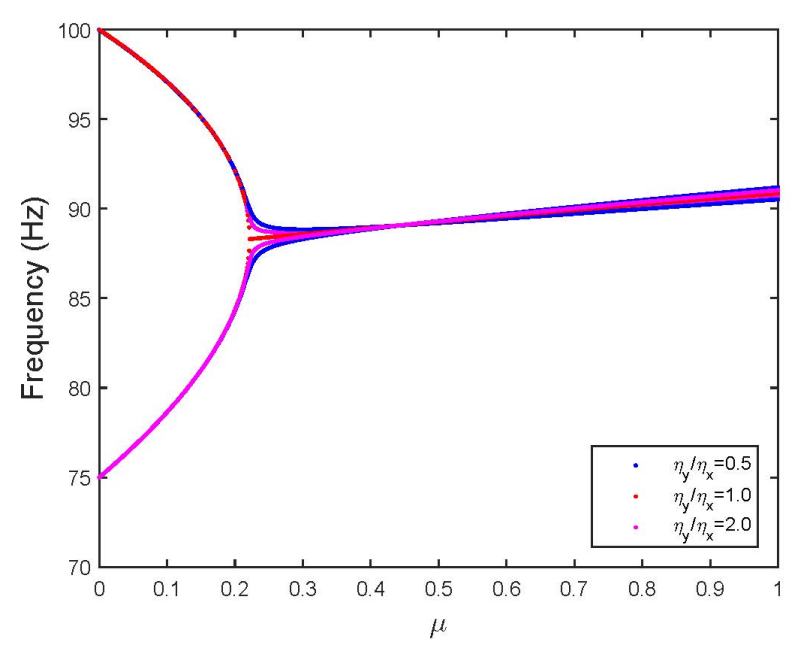

(f)

Figure 8: Influence of non-iso damping on (a,c,e) the real parts and (b,d,f) the frequencies with $\eta_{y}$ constant for $(\mathrm{a}, \mathrm{b})$ planar friction and $\theta=-30^{\circ},(\mathrm{c}, \mathrm{d})$ planar friction and $-60^{\circ}$, (e,f) rectilinear friction and $\theta=-30^{\circ}$ 


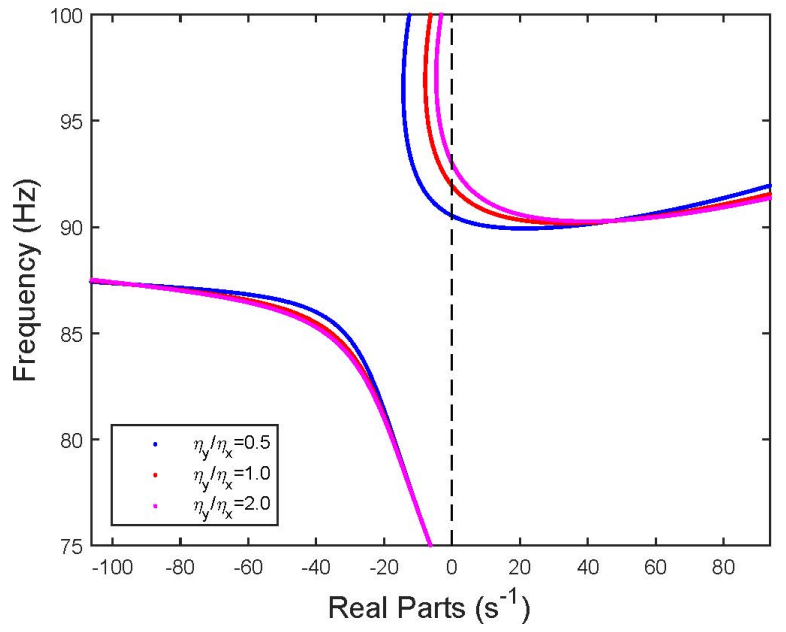

(a)

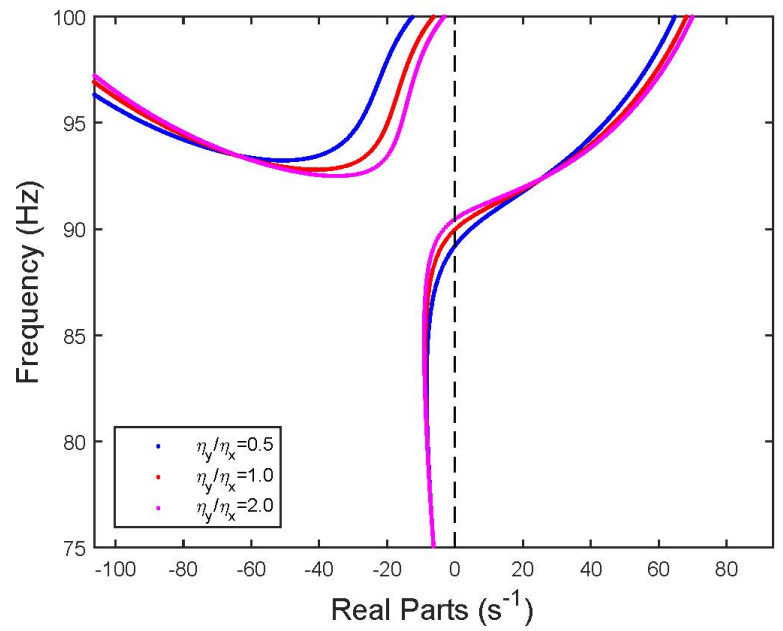

(b)

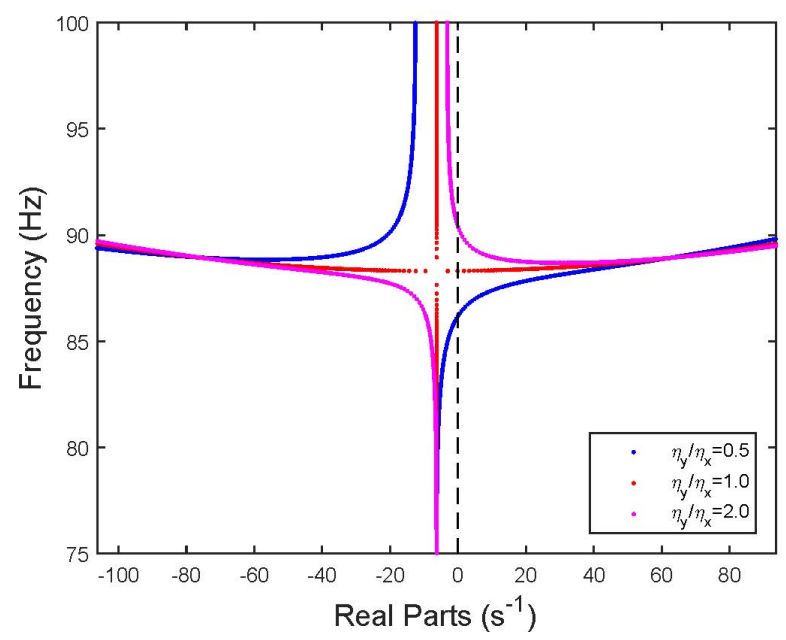

(c)

Figure 9: Influence of non-iso damping on the complex planes with $\eta_{y}$ constant for (a) planar friction and $\theta=-30^{\circ}$, (b) planar friction and $-60^{\circ}$ and (c) rectilinear friction and $\theta=-30^{\circ}$ 


\section{List of Tables}

1 Friction coefficient and unstable mode frequency at the Hopf bifurcation point for $\theta=-30^{\circ}$ (undamped

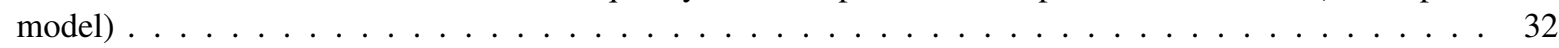

2 Friction coefficient and stable and unstable mode frequencies at the Hopf bifurcation point for planar friction, $\theta=-30^{\circ}$ and different sliding velocities (undamped model) . . . . . . . . . . . . . 33

3 Friction coefficient and unstable mode frequency at the Hopf bifurcation point for $\theta=-30^{\circ}$ (iso-damped

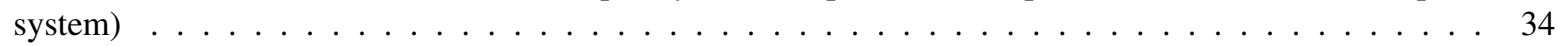

4 Friction coefficient and unstable mode frequency at the Hopf bifurcation point for $\eta_{y}=0.02$ (non-iso

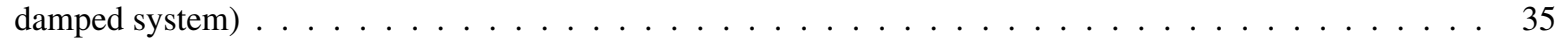




\begin{tabular}{|c|c|c|c|c|}
\hline$k_{1}-k_{2}$ & \multicolumn{2}{|c|}{ Planar Friction } & \multicolumn{2}{c|}{ Rectilinear Friction } \\
\cline { 2 - 5 }$\left(\mathrm{N}_{\mathrm{m}}^{-1}\right)$ & $\mu_{c}$ & unstable frequency $(\mathrm{Hz})$ & $\mu_{c}$ & unstable frequency $(\mathrm{Hz})$ \\
\hline $8.75 \times(2 \pi)^{2}$ & 0.029 & 89.12 & 0.043 & 87.81 \\
$43.75 \times(2 \pi)^{2}($ reference $)$ & 0.168 & 94.22 & 0.222 & 88.31 \\
$78.75 \times(2 \pi)^{2}$ & 0.347 & 96.44 & 0.412 & 87.58 \\
\hline
\end{tabular}

Table 1: Friction coefficient and unstable mode frequency at the Hopf bifurcation point for $\theta=-30^{\circ}$ (undamped model) 


\begin{tabular}{|c|c|c|c|}
\hline Sliding velocity $\left(\mathrm{m} . \mathrm{s}^{-1}\right)$ & $\mu_{c}$ & unstable frequency $(\mathrm{Hz})$ & stable frequency $(\mathrm{Hz})$ \\
\hline 1.0 & 0.168 & 94.24 & 81.02 \\
5.0 (reference) & 0.168 & 94.22 & 81.97 \\
10.0 & 0.168 & 94.22 & 82.00 \\
\hline
\end{tabular}

Table 2: Friction coefficient and stable and unstable mode frequencies at the Hopf bifurcation point for planar friction, $\theta=-30^{\circ}$ and different sliding velocities (undamped model) 


\begin{tabular}{|c|c|c|c|}
\hline Friction & damping & $\mu_{c}$ & unstable frequency $(\mathrm{Hz})$ \\
\hline Planar & $\eta=0.01$ & 0.195 & 92.70 \\
Planar & $\eta=0.02$ & 0.209 & 91.88 \\
Planar & $\eta=0.05$ & 0.236 & 90.74 \\
Rectilinear & $\eta=0.01$ & 0.222 & 88.27 \\
Rectilinear & $\eta=0.02$ & 0.224 & 88.28 \\
Rectilinear & $\eta=0.05$ & 0.231 & 88.31 \\
\hline
\end{tabular}

Table 3: Friction coefficient and unstable mode frequency at the Hopf bifurcation point for $\theta=-30^{\circ}$ (iso-damped system) 


\begin{tabular}{|c|c|c|c|c|}
\hline Velocity direction & Friction & Damping ratio $\eta_{y} / \eta_{x}$ & $\mu_{c}$ & unstable frequency $(\mathrm{Hz})$ \\
\hline$\theta=-30^{\circ}$ & Planar & 0.5 & 0.227 & 90.50 \\
$\theta=-30^{\circ}$ & Planar & 1.0 & 0.209 & 91.88 \\
$\theta=-30^{\circ}$ & Planar & 2.0 & 0.192 & 93.00 \\
$\theta=-30^{\circ}$ & Rectilinear & 0.5 & 0.219 & 86.30 \\
$\theta=-30^{\circ}$ & Rectilinear & 1.0 & 0.224 & 88.28 \\
$\theta=-30^{\circ}$ & Rectilinear & 2.0 & 0.216 & 90.39 \\
$\theta=-60^{\circ}$ & Planar & 0.5 & 0.206 & 89.30 \\
$\theta=-60^{\circ}$ & Planar & 1.0 & 0.208 & 89.98 \\
$\theta=-60^{\circ}$ & Planar & 2.0 & 0.209 & 90.49 \\
\hline
\end{tabular}

Table 4: Friction coefficient and unstable mode frequency at the Hopf bifurcation point for $\eta_{y}=0.02$ (non-iso damped system) 